\title{
Derivation of All Linear Transformations that Meet the Results of Michelson-Morley's Experiment and Discussion of the Relativity Basics
}

\author{
Roman Szostek \\ Rzeszow University of Technology, Department of Quantitative Methods, Rzeszow, Poland \\ rszostek@prz.edu.pl
}

\begin{abstract}
:
The paper presents a formal proof that the mathematics on which the Special Theory of Relativity (STR) is based is currently misinterpreted. The evidence is based on an analysis of the importance of parameter $e(v)$. Understanding the meaning of this parameter was achieved by analyzing the general form of transformation, for which the Lorentz transformation is only a special case. If $e(v) \neq 0$ then the clocks in inertial systems are desynchronized. Measurements, e.g. one-way speed, using such clocks do not give real values.

The article shows that there are infinitely many different transformations in which one-way speed of light is always equal to $c$. The Lorentz transformation is only one of those infinitely many transformations.

In this article, the whole class of linear transformations of time and coordinate was derived. Transformations were derived on the assumption that conclusions from Michelson-Morley's and Kennedy-Thorndikea's experiments are met for the observer from each inertial frame of reference, i.e. that the mean velocity of light in the vacuum flowing along the way back and forth is constant. It was also assumed that there is at least one inertial frame of reference, in which the velocity of light in a vacuum in each direction has the same value $c$, and the space is isotropic for observers from this distinguished inertial frame of reference (universal frame of reference).

Derived transformations allow for building many different kinematics according to Michelson-Morley's and Kennedy-Thorndikea's experiments.

The class of transformations derived in the study is a generalization of transformations derived in the paper [10], which consists in enabling non-zero values of parameter $e(v)$. The idea of such a generalization derives from the person, who gave me this extended transformations class for analysis and publication.
\end{abstract}

Keywords: coordinate and time transformation; kinematics; universal frame of reference; one-way speed of light; anisotropy of cosmic microwave background

\section{Introduction}

The class of transformations derived in this article is a generalization of transformations derived in the paper [10]. In that paper all linear transformations that are possible for the parameter $e(v)=0$ were derived. In the paper [11] one of these transformations was analyzed. 
Derivation of all linear transformations that meet the results of Michelson-Morley's experiment and discussion of the relativity basics - Szostek Roman

It is a common belief in the contemporary physics that the Michelson-Morley [3] and Kennedy-Thorndike [1] experiments proved that the velocity of light is absolutely constant and that there is no universal frame of reference called the ether. Based on the analysis of these experiments, the Lorentz transformation, on which the Special Theory of Relativity is based, was derived. It is currently considered that the Special Theory of Relativity is the only theory of kinematics of bodies which correctly explains the Michelson-Morley experiment and all other experiments in which the velocity of light is measured.

It turns out that the velocity of light in one direction (momentary) has never been accurately measured. In all accurate measurements of the velocity of light, only the average velocity of light traveling the path along the closed trajectory was measured. In order to measure the velocity of light, light had to return to the measuring device. In the simplest case, light was sent to a mirror and back as was done in experiments by Armand Fizeau in 1849 and by Jean Foucault in 1850. The same happens in Michelson-Morley and Kennedy-Thorndike experiments in which sources of light after being reflected by mirrors return to the source point. From these experiments, it is clear that the average velocity of light traveling the path to and back is constant, and not that the velocity of light in one direction (momentary) is constant.

There are publications in which numerous coordinate and time transformations [2], [4], [5], [6], [14] are presented. In this article all possible linear transformations (without turnover) are derived. Derivation presented in this article is based on the postulate of average velocity of light, and not on clock synchronization. From the presented analysis it results that there are infinitely many coordinates and time transformations which are in accordance with the results of MichelsonMorley's experiment. On the basis of these transformations many kinematics of bodies can be built, describing different physical properties, such as time dilation. It follows that there are infinitely many different kinematics, which are consistent with the results of Michelson-Morley's experiments.

\section{Adopted assumptions}

The following assumptions have been adopted in the presented analysis:

I. Coordinate and time transformation «inertial frame of reference - inertial frame of reference» is linear.

II. There is at least one inertial frame of reference in which the velocity of light in a vacuum is the same in each direction. This system is called a universal frame of reference. This one-way speed of light constant is indicated by the $\operatorname{symbol} c=$ constants.

III. The average velocity of light in the vacuum flowing way back and forth is constant for each observer from the inertial frame of reference. This average velocity does not depend on the observer's velocity in relation to the universal frame of reference, nor on the direction of light propagation. This average velocity is indicated by the symbol $c_{p}$.

On the basis of assumption II and III it can be shown that the average velocity $c_{p}$ is equal to one-way speed $c$. It is important to note that on the basis of III, the $c_{p}$ value is the same for each observer, i.e. also for the one that does not move in relation to the universal frame of reference. As for the motionless observer in relation to the universal frame of reference it has the value of $c$, thus $c_{p}=c$.

Let the light impulse move along a path of length $L$ in one direction with velocity $c^{+} \geq 0$ at time $t_{1}$, and in other direction along the same path $L$ with velocity $c^{-} \leq 0$ at time $t_{2}$. Then the average velocity of light on the back and forth path is 
Derivation of all linear transformations that meet the results of Michelson-Morley's experiment and discussion of the relativity basics - Szostek Roman

$$
c_{p}=c=\frac{2 L}{t_{1}+t_{2}}=\frac{2 L}{\frac{L}{c^{+}}+\frac{L}{-c^{-}}}=\frac{2}{\frac{1}{c^{+}}-\frac{1}{c^{-}}}
$$

On this basis, an assumption III in the form of following equation is obtained

$$
\frac{1}{c^{+}}-\frac{1}{c^{-}}=\frac{2}{c}
$$

\section{Transformations for one spatial dimension}

Indications shown in Figure 1 are adopted. Coordinates in the universal frame of reference $U$ will be indicated with symbols $x, t$. Coordinates in the inertial frame of reference $U^{\prime}$ will be indicated with symbols $x^{\prime}, t^{\prime}$. The inertial frame of reference $U^{\prime}$ moves in relation to the universal frame of reference $U$ with velocity $v$ along parallel $x$ and $x^{\prime}$ axes. All velocities with direction such as $x$ axis direction (or $x^{\prime}$ in $U^{\prime}$ frame) have positive values, while those with opposite direction have negative values. But the symbol $c$ will always have a positive value, regardless of the direction in which the light moves, i.e. always $c=+299792458 \mathrm{~m} / \mathrm{s}$.

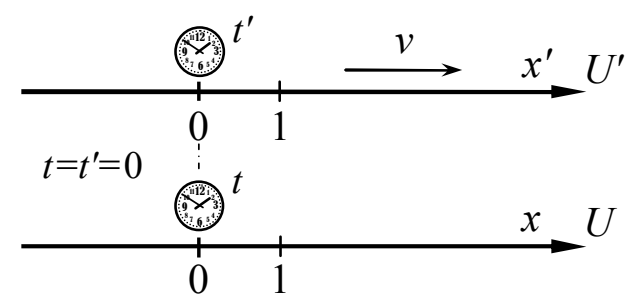

Fig. 1. Inertial frame of reference $U^{\prime}$ move in relation to the universal frame of reference $U$ with velocity $v$.

When the beginnings of frames overlapped, then the clocks at that beginning were reset to zero. The clocks in universal frame of reference $U$ were synchronized with the clock at the beginning of this system with the light of Einstein method. At this stage, it is not determined how the clocks in $U^{\prime}$ frame are synchronized.

Transformation from a universal frame of reference $U$ to an inertial frame of reference $U^{\prime}$ has, on the basis of assumption I, the following form

$$
\left\{\begin{array}{l}
x^{\prime}=a x+b t \\
t^{\prime}=e_{1} x+f t
\end{array}\right.
$$

Transformation parameters are continuous velocity $v$ functions with the following properties

$$
\begin{array}{llll}
a(0)=1 & \wedge & a(v)>0 & \\
b(0)=0 & \wedge \quad(v>0 \Rightarrow b(v)<0) \wedge(v<0 \Rightarrow b(v)>0) & {[\mathrm{m} / \mathrm{s}]} \\
e_{1}(0)=0 & & & {[\mathrm{~s} / \mathrm{m}]} \\
f(0)=1 & \wedge & f(v)>0 & {[1]}
\end{array}
$$

Parameters $a(0)=1, b(0)=0, e_{1}(0)=0$ and $f(0)=1$ because for $v=0$ the systems $U$ and $U^{\prime}$ are identical, i.e. they show the same coordinates of position and time.

The condition $a(v)>0$ must be met due to the same direction of $x$ and $x^{\prime}$ axes (Figure 1), i.e. if $x$ increases, then $x^{\prime}$ also increases. The condition $(v>0 \Rightarrow b(v)<0)$ must be met due to the same direction of $x$ and $x^{\prime}$ axes (Figure 1), i.e. for established $x$ coordinate if time $t$ elapse, then $x^{\prime}$ coordinate decreases. Similarly, if the velocity $v$ is negative, i.e. the $U^{\prime}$ frame moves in the opposite 
Derivation of all linear transformations that meet the results of Michelson-Morley's experiment and discussion of the relativity basics - Szostek Roman

direction, then coordinate $x^{\prime}$ increases, i.e. $(v<0 \Rightarrow b(v)>0)$. Condition $f(v)>0$ must be met as if $t$ increases, $t^{\prime}$ increases as well.

For our needs it will conveniently use the parameter $e(v)$, where $e_{1}(v)=v \cdot e(v)$. Introduction of such a parameter is acceptable, because according to (4) there is an $e_{1}(0)=0$. The parameter $e(v)$ $\left[\mathrm{s}^{2} / \mathrm{m}^{2}\right]$ is a continuous velocity function $v$. Now the transformation (3) takes a form of

$$
\left\{\begin{array}{l}
x^{\prime}=a x+b t \\
t^{\prime}=e v x+f t
\end{array}\right.
$$

Differentials from transformation (5) have the form ( $v=$ constants) of

$$
\left\{\begin{array}{l}
d x^{\prime}=a d x+b d t \\
d t^{\prime}=e v d x+f d t
\end{array}\right.
$$

Let's consider the body, which rests in the inertial frame of reference $U^{\prime}$. As it is motionless in this system, and therefore for its coordinate of position there is

$$
d x^{\prime}=0
$$

Note that the velocity of body under consideration in relation to system $U$ (i.e. $d x / d t$ ) is a velocity $v$ of system $U^{\prime}$ in relation to system $U$. Therefore

$$
\frac{d x}{d t}=v
$$

From the differential coordinate (6) on the basis of (7) and (8) the following is obtained

$$
0=d x^{\prime}=\frac{d x^{\prime}}{d t}=\frac{a d x+b d t}{d t}=a \frac{d x}{d t}+b=a v+b
$$

It follows that

$$
b=-a v
$$

On this basis the transformation (5) takes the form of

$$
\left\{\begin{array}{l}
x^{\prime}=a(x-v t) \\
t^{\prime}=e v x+f t
\end{array}\right.
$$

Differentials from transformation (11) have the form ( $v=$ constants) of

$$
\left\{\begin{array}{l}
d x^{\prime}=a(d x-v d t) \\
d t^{\prime}=e v d x+f d t
\end{array}\right.
$$

Now consider a light impulse that moves along $x$ and $x^{\prime}$ axes. When light moves in the direction of $x^{\prime}$ axis and velocity $v$, then the velocity of light in the inertial frame of reference $U^{\prime}$ has the following value

$$
\frac{d x^{\prime}}{d t^{\prime}}=c_{x}^{+}(v)
$$

while in the universal frame of reference $U$ has a value (assumption II) of

$$
\frac{d x}{d t}=c \geq 0
$$


Derivation of all linear transformations that meet the results of Michelson-Morley's experiment and discussion of the relativity basics - Szostek Roman

When light moves in the direction opposite to $x^{\prime}$ axis and velocity $v$, then the velocity of light in the inertial frame of reference $U^{\prime}$ has the value of

$$
\frac{d x^{\prime}}{d t^{\prime}}=c_{x}^{-}(v)
$$

while in the universal frame of reference $U$ has a value (assumption II) of

$$
\frac{d x}{d t}=-c \leq 0
$$

When the differentials (12) are divided into sides, then on the basis of (13) and (14) the following is obtained

$$
c_{x}^{+}(v)=\frac{d x^{\prime}}{d t^{\prime}}=\frac{a(d x-v d t)}{e v d x+f d t}=\frac{a\left(\frac{d x}{d t}-v\right)}{e v \frac{d x}{d t}+f}=\frac{a(c-v)}{e v c+f}
$$

When the differentials (12) are divided into sides, then on the basis of (15) and (16) the following is obtained

$$
c_{x}^{-}(v)=\frac{d x^{\prime}}{d t^{\prime}}=\frac{a(d x-v d t)}{e v d x+f d t}=\frac{a\left(\frac{d x}{d t}-v\right)}{e v \frac{d x}{d t}+f}=\frac{-a(c+v)}{-e v c+f}
$$

Formula (18) can be obtained from formula (17) by changing the sign before velocity $c$ (this means changing the direction of movement of a light pulse).

Formula (18) can also be obtained by simultaneously changing (17) the signs before velocities $v$ and $c_{x}^{+}$(this means changing the direction of velocities $v$ and $x^{\prime}$ axis direction at the same time). In order to obtain formula (18), parameter $e(v)$ must not change the sign. On this basis the following properties are obtained for this parameter

$$
e(v) \cdot e(-v) \geq 0
$$

From formulas (17) and (18) after taking into account (19), it results that one-way speed of light functions meet the following relation

$$
\begin{gathered}
c_{x}^{-}(v, c)=-c_{x}^{+}(-v, c) \\
c_{x}^{-}(v, c)=c_{x}^{+}(v,-c)
\end{gathered}
$$

If dependencies (17) and (18) are added to equation (2) then it is obtained as follows

$$
\frac{e v c+f}{a(c-v)}+\frac{-e v c+f}{a(c+v)}=\frac{2}{c}
$$

In order for condition (2) to be met, parameter $a(v)$ must be in the form of

$$
a=c^{2} \cdot \frac{f+e v^{2}}{c^{2}-v^{2}}=\frac{1}{1-(v / c)^{2}}\left(f+e v^{2}\right)=\gamma^{2}\left(f+e v^{2}\right)
$$

On the basis of (23) the transformation (11) takes the form of 
Derivation of all linear transformations that meet the results of Michelson-Morley's experiment and discussion of the relativity basics - Szostek Roman

$$
\left\{\begin{array}{l}
x^{\prime}=\gamma^{2}\left(f+e v^{2}\right)(x-v t) \\
t^{\prime}=e v x+f t
\end{array}\right.
$$

On this basis it is possible to determine the reverse transformation from the inertial frame of reference $U^{\prime}$ to the universal frame of reference $U$ in the form of

$$
\left\{\begin{array}{l}
x=\frac{f}{\gamma^{2}\left(f+e v^{2}\right)^{2}} x^{\prime}+\frac{1}{f+e v^{2}} v t^{\prime} \\
t=\frac{-e v}{\gamma^{2}\left(f+e v^{2}\right)^{2}} x^{\prime}+\frac{1}{f+e v^{2}} t^{\prime}
\end{array}\right.
$$

Equations (24) and (25) are transformations sought for one spatial dimension. They contain two parameters $e(v)$ and $f(v)$. These parameters must meet conditions (4) and (19). After the adoption of specific parameters, a specific transformation is obtained describing the specific kinematics. This general form of transformation contains all possible linear transformations between the universal frame of reference $U$, in which light propagates with the constant velocity $c$, and the inertial frame of reference $U^{\prime}$ moving relative to system $U$ with velocity $v$, along $x$ and $x^{\prime}$ axes, if in the inertial frame of reference $U^{\prime}$ of one-way speed of light has met condition (2) (i.e. assumption III).

\section{Transformations for three spatial dimensions}

In order to introduce two remaining spatial dimensions to the transformation, additional assumption will be adopted:

IV. For each motionless observer in relation to the universal frame of reference, the space is isotropic, i.e. it has the same properties in each direction.

From assumption IV, results that parameters occurring in transformation (6) and (24)-(25) meet the following properties

$$
\begin{aligned}
& a(v)=a(-v) \\
& b(v)=-b(-v) \\
& e(v)=e(-v) \\
& f(v)=f(-v)
\end{aligned}
$$

Properties (26) result from (4), (19) and from the following reasoning. Parameters $a(v)$ and $f(v)$ must be even functions as if $x^{\prime}$ increases, then $x^{\prime}$ increases and if $t$ increases then $t^{\prime}$ increases, the same applies regardless of the direction of velocities $v$. The parameter $b(v)$ must be odd function, because after a change of velocities $v$ direction for a fixed coordinate $x$, if time $t$ elapses, the coordinate $x^{\prime}$ increases just as it did decreases for unchanged velocities $v$ direction. The parameter $v \cdot e(v)$ must be odd because after a change of direction of velocities $v$ for a fixed time $t$, the change of time $t^{\prime}$ depends on $x$ in the opposite way to unchanged direction of velocities $v$. Therefore, the parameter $e(v)$ must be even function.

Consider the situation shown in Figure 2. In the inertial frame of reference $U^{\prime}$, the light impulse moves perpendicularly to $x^{\prime}$ axis. This light passes a distance of $L^{\prime}$, first in one direction and then back, i.e. it returns to the starting point.

Due to the assumption IV, the velocity of light in a direction perpendicular to $x^{\prime}$ axis is the same in one direction and in the other and is $c$. This results from the fact that no direction 
Derivation of all linear transformations that meet the results of Michelson-Morley's experiment and discussion of the relativity basics - Szostek Roman

perpendicular to velocities $v$ (i.e. also $x$ and $x^{\prime}$ axes) is distinguished (assumption IV) and the average velocity of light on the back and forth path is $c$ (assumption III). For this reason, the same light impulse for the motionless observer in relation to the universal frame of reference $U$ will move along the arms of an isosceles triangle. For an observer from the system $U$, the dimensions perpendicular to velocities $v$ may be different than for an observer from the $U^{\prime}$ system, and therefore the height of triangle is determined by

$$
L=\psi(v) L^{\prime}
$$

The parameter $\psi(v)$ describes the transverse contraction of bodies moving in relation to the universal frame of reference. This parameter should meet the following conditions

$$
\psi(0)=1 \wedge \psi(v)>0 \quad[1]
$$

The parameter $\psi(0)=1$ as for $v=0$, the transverse dimensions are identical for observers from $U$ and $U^{\prime}$ systems. Condition $\psi(v)>0$ must be fulfilled because the transverse dimensions do not reverse.
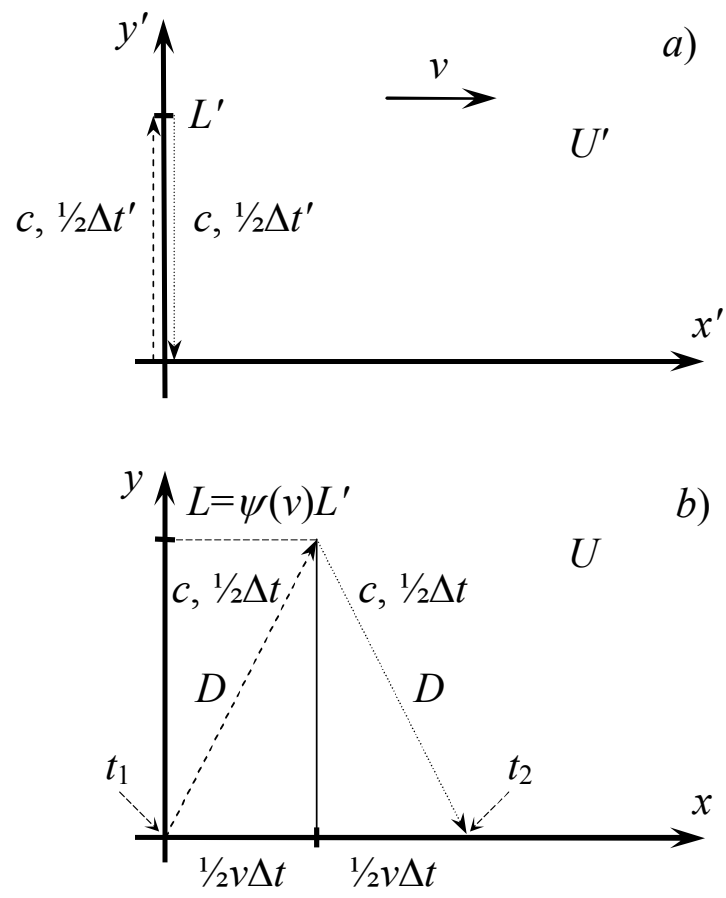

Fig. 2. The path of light seen from two frames of reference.

a) inertial frame of reference $\left.U^{\prime}, b\right)$ universal frame of reference $U$.

Due to assumption IV for the observer from the system $U$, the transverse dimensions are contracted in the same way for each direction of velocities $v$. Therefore, parameter $\psi(v)$ should meet the following condition

$$
\psi(v)=\psi(-v)
$$

Now the parameter $\psi(v)$ will be set.

For an observer from the system $U^{\prime}$, the following occurs

$$
\Delta t^{\prime}=\frac{2 L^{\prime}}{c} \Leftrightarrow L^{\prime}=\frac{c \Delta t^{\prime}}{2}
$$

On the basis of transformation (25) the differential ( $v=$ constants) is obtained. 
Derivation of all linear transformations that meet the results of Michelson-Morley's experiment and discussion of the relativity basics - Szostek Roman

$$
d t=\frac{-e v}{\gamma^{2}\left(f+e v^{2}\right)^{2}} d x^{\prime}+\frac{1}{f+e v^{2}} d t^{\prime}
$$

I.e. for the fixed coordinate $x^{\prime}$ in the system $U^{\prime}$ it is obtained as follows

$$
d x^{\prime}=0 \Rightarrow d t=\frac{1}{f+e v^{2}} d t^{\prime}
$$

Formula (32) describes time dilation for motionless clock relative to $U^{\prime}$. In Figure 2 such a clock is at the beginning of system $U^{\prime}$. If the time $\Delta t^{\prime}$ on this clock elapses, appearing in formula (30), then in system $U$ will elapse the time $\Delta t=t_{2}-t_{1}$, where $t_{1}$ is the time at which an impulse was sent, and $t_{2}$ is the time when an impulse returned to $x$ axis. Times $t_{1}$ and $t_{2}$ are measured in system $U$ by two different clocks. According to formula (32), the following occurs

$$
\Delta t=\frac{1}{f+e v^{2}} \Delta t^{\prime}
$$

From the geometry of figure it is obtained as follows

$$
D=\sqrt{v^{2} \Delta t^{2} / 4+\psi^{2} L^{\prime 2}}
$$

and

$$
\Delta t=\frac{2 D}{c}
$$

From equations (34) and (35) it is obtained as follows

$$
\begin{gathered}
\Delta t=\frac{2 \sqrt{v^{2} \Delta t^{2} / 4+\psi^{2} L^{\prime 2}}}{c} \\
c^{2} \Delta t^{2}=4\left(v^{2} \Delta t^{2} / 4+\psi^{2} L^{\prime 2}\right) \\
4 \psi^{2} L^{\prime 2}=\left(c^{2}-v^{2}\right) \Delta t^{2}
\end{gathered}
$$

On the basis of (30) and (33) it is obtained as follows

$$
\begin{gathered}
4 \psi^{2} \frac{c^{2} \Delta t^{\prime 2}}{4}=\left(c^{2}-v^{2}\right) \frac{1}{\left(f+e v^{2}\right)^{2}} \Delta t^{\prime 2} \\
\psi^{2}=\frac{c^{2}-v^{2}}{c^{2}} \frac{1}{\left(f+e v^{2}\right)^{2}} \\
\psi=\sqrt{1-(v / c)^{2}} \frac{1}{f+e v^{2}}=\frac{1}{\frac{1}{\sqrt{1-(v / c)^{2}}}\left(f+e v^{2}\right)}
\end{gathered}
$$

That is, the transverse contraction parameter $\psi(v)$ must have the following value

$$
\psi=\frac{1}{\gamma\left(f+e v^{2}\right)}
$$

The above analysis shows that formula (42) per parameter $\psi(v)$ results from the assumption IV and time dilation (32). 
Derivation of all linear transformations that meet the results of Michelson-Morley's experiment and discussion of the relativity basics - Szostek Roman

After taking into account (42) in transformation (24)-(25), the transformations for three spatial dimensions described by parameters $e(v)$ and $f(v)$ are obtained. From the universal frame of reference $U$ to inertial frame of reference $U^{\prime}$ has the following form

$$
\left\{\begin{array}{l}
t^{\prime}=e v x+f t \\
x^{\prime}=\gamma^{2}\left(f+e v^{2}\right)(x-v t) \\
y^{\prime}=\gamma\left(f+e v^{2}\right) y \\
z^{\prime}=\gamma\left(f+e v^{2}\right) z
\end{array}\right.
$$

From the inertial frame of reference $U^{\prime}$ to the universal frame of reference $U$ has the following form

$$
\left\{\begin{array}{l}
t=\frac{-e v}{\gamma^{2}\left(f+e v^{2}\right)^{2}} x^{\prime}+\frac{1}{f+e v^{2}} t^{\prime} \\
x=\frac{f}{\gamma^{2}\left(f+e v^{2}\right)^{2}} x^{\prime}+\frac{1}{f+e v^{2}} v t^{\prime} \\
y=\frac{1}{\gamma\left(f+e v^{2}\right)} y^{\prime} \\
z=\frac{1}{\gamma\left(f+e v^{2}\right)} z^{\prime}
\end{array}\right.
$$

The relation (42) can be written in a different way

$$
f=\frac{1}{\gamma \psi}-e v^{2}
$$

After taking into account (45) in transformation (24)-(25) or (43)-(44), the transformations for three spatial dimensions described by parameters $e(v)$ and $\psi(v)$ are obtained. From the universal frame of reference $U$ to inertial frame of reference $U^{\prime}$ the transformation has the following form

$$
\left\{\begin{array}{l}
t^{\prime}=e v x+\left(\frac{1}{\gamma \psi}-e v^{2}\right) t \\
x^{\prime}=\frac{\gamma}{\psi}(x-v t) \\
y^{\prime}=\frac{1}{\psi} y \\
z^{\prime}=\frac{1}{\psi} z
\end{array}\right.
$$

From the inertial frame of reference $U^{\prime}$ to the universal frame of reference $U$, the transformation has the form

$$
\left\{\begin{array}{l}
t=-\psi^{2} e v x^{\prime}+\gamma \psi t^{\prime} \\
x=\left(\frac{\psi}{\gamma}-\psi^{2} e v^{2}\right) x^{\prime}+\gamma \psi v t^{\prime} \\
y=\psi y^{\prime} \\
z=\psi z^{\prime}
\end{array}\right.
$$


Derivation of all linear transformations that meet the results of Michelson-Morley's experiment and discussion of the relativity basics - Szostek Roman

Equations (43)-(44) and (46)-(47) are transformations sought for all spatial dimensions. The transformation set (43)-(44) is identical to the transformation set (46)-(47). These sets differ only in the parameters used.

\section{Selected transformations properties}

Differentials from transformation (46) have the form ( $v=$ constants) of

$$
\left\{\begin{array}{l}
d t^{\prime}=e v d x+\left(\frac{1}{\gamma \psi}-e v^{2}\right) d t \\
d x^{\prime}=\frac{\gamma}{\psi}(d x-v d t) \\
d y^{\prime}=\frac{1}{\psi} d y \\
d z^{\prime}=\frac{1}{\psi} d z
\end{array}\right.
$$

Differentials from transformation (47) have the form ( $v=$ constants) of

$$
\left\{\begin{array}{l}
d t=-\psi^{2} e v d x^{\prime}+\gamma \psi d t^{\prime} \\
d x=\left(\frac{\psi}{\gamma}-\psi^{2} e v^{2}\right) d x^{\prime}+\gamma \psi v d t^{\prime} \\
d y=\psi d y^{\prime} \\
d z=\psi d z^{\prime}
\end{array}\right.
$$

\subsection{Time dilation}

The formula for time dilation for (46)-(47) transformation will be determined.

From the differential time (48) results that for the motionless observer in relation to universal frame of reference $U$, the following formula for time dilation is given (also based on (45))

$$
d x=0 \Rightarrow d t^{\prime}=\left(\frac{1}{\gamma \psi}-e v^{2}\right) d t=f(v) d t
$$

From the differential time (49) results that for the motionless observer in relation to inertial frame of reference $U^{\prime}$, the following formula for time dilation is given

$$
d x^{\prime}=0 \Rightarrow d t=\gamma \psi d t^{\prime}
$$

From formulas(50) and (51) results that observers from frames of reference $U$ and $U^{\prime}$ moving relative to each other will measure the same time dilation only if the parameter $e(v)=0$. If $e(v) \neq 0$ then these two observers evaluate the relative passage of time differently on compared clocks.

Time dilation (50) and (51) is written as an implication, as it is more precise than the record commonly used in physics. 
Derivation of all linear transformations that meet the results of Michelson-Morley's experiment and discussion of the relativity basics - Szostek Roman

\subsection{Longitudinal length contraction (Lorentz-Fitz-Gerald)}

The formulas for longitudinal length contraction (along $x$ and $x^{\prime}$ axes) for transformation (46)-(47) are determined.

From the differential coordinate (48), the following formula for longitudinal length contraction (also based on (23) and (45)) results for the observer from the universal frame of reference $U$

$$
d t=0 \Rightarrow d x^{\prime}=\frac{\gamma}{\psi} d x=a(v) d x
$$

From the differential coordinate (49), the following formula for longitudinal length contraction results for the observer from the universal frame of reference $U$

$$
d t^{\prime}=0 \Rightarrow d x=\left(\frac{\psi}{\gamma}-\psi^{2} e v^{2}\right) d x^{\prime}
$$

From formulas (52) and (53) results that observers from frames of reference $U$ and $U^{\prime}$ moving relative to each other will measure the same longitudinal contraction only if the parameter $e(v)=0$. If $e(v) \neq 0$ then these two observers evaluate differently the proportions of the longitudinal dimensions measured by them.

Longitudinal length contraction (52) and (53) is written as implications, as this is more precise than what is commonly used in physics.

\subsection{Transformations of velocity}

The formulas for transformations of velocity for transformations (46)-(47) will be determined. Indications as in Figure 3 are adopted. The body moves in relation to $U$ and $U^{\prime}$ systems. For an observer from the system $U$ it has velocity $V$, while for an observer from the system $U^{\prime}$ it has velocity $V^{\prime}$.

From equations (48) the following equations result

$$
\left\{\begin{array}{l}
\frac{d x^{\prime}}{d t^{\prime}}=\frac{\frac{\gamma}{\psi}(d x-v d t)}{e v d x+\left(\frac{1}{\gamma \psi}-e v^{2}\right) d t} \\
\frac{d y^{\prime}}{d t^{\prime}}=\frac{\frac{1}{\psi} d y}{e v d x+\left(\frac{1}{\gamma \psi}-e v^{2}\right) d t} \\
\frac{d z^{\prime}}{d t^{\prime}}=\frac{\frac{1}{\psi} d z}{e v d x+\left(\frac{1}{\gamma \psi}-e v^{2}\right) d t}
\end{array}\right.
$$

On this basis, the transformation of velocities from system $U$ to system $U^{\prime}$ has the form of 


$$
\left\{\begin{array}{l}
V_{x}^{\prime}=\frac{\gamma^{2}\left(V_{x}-v\right)}{\gamma \psi e v\left(V_{x}-v\right)+1} \\
V_{y}^{\prime}=\frac{\gamma V_{y}}{\gamma \psi e v\left(V_{x}-v\right)+1} \\
V_{z}^{\prime}=\frac{\gamma V_{z}}{\gamma \psi e v\left(V_{x}-v\right)+1}
\end{array}\right.
$$
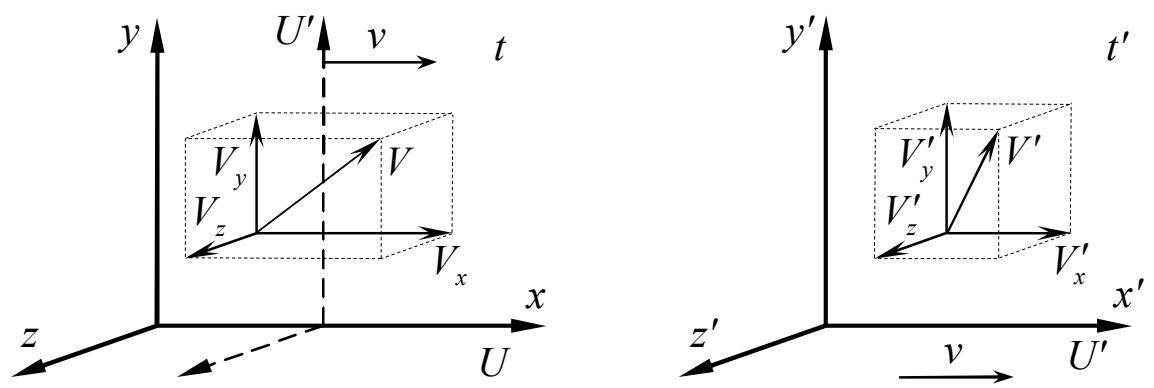

Fig. 3. Movement seen from the universal frame of reference and inertial frame of reference.

From time equation (49) the following equations result

$$
\left\{\begin{array}{l}
\frac{d x}{d t}=\frac{\left(\frac{\psi}{\gamma}-\psi^{2} e v^{2}\right) d x^{\prime}+\gamma \psi v d t^{\prime}}{-\psi^{2} e v d x^{\prime}+\gamma \psi d t^{\prime}} \\
\frac{d y}{d t}=\frac{\psi d y^{\prime}}{-\psi^{2} e v d x^{\prime}+\gamma \psi d t^{\prime}} \\
\frac{d z}{d t}=\frac{\psi d z^{\prime}}{-\psi^{2} e v d x^{\prime}+\gamma \psi d t^{\prime}}
\end{array}\right.
$$

On this basis, the transformation of velocities from system $U^{\prime}$ to system $U$ has the form of

$$
\left\{\begin{array}{l}
V_{x}=\frac{\left(\frac{\psi}{\gamma}-\psi^{2} e v^{2}\right) V_{x}^{\prime}+\gamma \psi v}{-\psi^{2} e v V_{x}^{\prime}+\gamma \psi} \\
V_{y}=\frac{\psi V_{y}^{\prime}}{-\psi^{2} e v V_{x}^{\prime}+\gamma \psi} \\
V_{z}=\frac{\psi V_{z}^{\prime}}{-\psi^{2} e v V_{x}^{\prime}+\gamma \psi}
\end{array}\right.
$$

Transformations of velocity (55) and (57) are equivalent. It is possible to show that after introducing one to the other, the identity equations are obtained.

\subsection{Velocity of light along axis $x^{\prime}$ seen in the inertial frame of reference}

If the body shown in Figure 3 is an impulse of light, it moves with velocity $c$ in system $U$. Let's consider only the case in which this impulse moves parallel to $x$ and $x^{\prime}$ axes (i.e. it also moves parallel to velocities $v$ ). Then 
Derivation of all linear transformations that meet the results of Michelson-Morley's experiment and discussion of the relativity basics - Szostek Roman

$$
V_{x}=c, \quad V_{y}=0, \quad V_{z}=0
$$

On the basis of transformations (55), the velocity of this impulse of light seen in the inertial frame of reference $U^{\prime}$ is obtained

$$
c_{x}^{+}(v)=V_{x}^{\prime}=\frac{\gamma^{2}(c-v)}{\gamma \psi \operatorname{ev}(c-v)+1}, \quad c_{y}^{\prime}=V_{y}^{\prime}=0, \quad c_{z}^{\prime}=V_{z}^{\prime}=0
$$

(45). Due to

Formula (59) can also be obtained from formula (17) after applying dependencies (23) and

$$
\gamma^{2}(c-v)=\frac{1}{1-(v / c)^{2}}(c-v)=\frac{c^{2}}{c^{2}-v^{2}}(c-v)=\frac{c^{2}}{(c+v)(c-v)}(c-v)=\frac{c^{2}}{c+v}
$$

therefore, on the basis of (59) one-way speed of light with the same direction as $x^{\prime}$ axis direction and velocities $v$ direction has in inertial frame of reference $U^{\prime}$ the value of

$$
c_{x}^{+}(v)=\frac{c^{2}}{\frac{\psi}{\gamma} e v c^{2}+c+v}, \quad c_{y}^{\prime}=0, \quad c_{z}^{\prime}=0
$$

One-way speed of light with the opposite direction to $x^{\prime}$ axis direction and velocities $v$ direction has a value in the inertial frame of reference $U^{\prime}$

$$
c_{x}^{-}(v)=\frac{c^{2}}{\frac{\psi}{\gamma} e v c^{2}-c+v}, \quad c_{y}^{\prime}=0, \quad c_{z}^{\prime}=0
$$

Formula (62) is based on Formula (61) by changing the sign before velocity $c$ (this means changing the direction of movement of a light impulse). It can also be obtained by changing in formula (61) the sign in front of velocity $v$ (dependence (20) should then be taken into account, which means a change in $x^{\prime}$ axis direction). Then before the parameters $\psi(v), \gamma(v)$ and $e(v)$, it is not necessary to change the sign as these are even functions. Precisely because of such situations, it is more convenient to use the even function $e(v)$ used in transformation (5) than the odd function $e_{1}(v)$ used in transformation (4).

\subsection{Conclusions on one-way speed of light and the parameter $e(v)$}

On the basis of (61) it is obtained

$$
e=\frac{\gamma}{\psi} \cdot \frac{c^{2}-c_{x}^{+}(c+v)}{v c^{2} c_{x}^{+}}
$$

On the basis of (62) it is obtained

$$
e=\frac{\gamma}{\psi} \cdot \frac{c^{2}+c_{x}^{-}(c-v)}{v c^{2} c_{x}^{-}}
$$

On this basis, it is obtained

$$
\frac{\gamma}{\psi} \cdot \frac{c^{2}+c_{x}^{-}(c-v)}{v c^{2} c_{x}^{-}}=\frac{\gamma}{\psi} \cdot \frac{c^{2}-c_{x}^{+}(c+v)}{v c^{2} c_{x}^{+}}
$$


Derivation of all linear transformations that meet the results of Michelson-Morley's experiment and discussion of the relativity basics - Szostek Roman

$$
\begin{gathered}
\frac{c^{2}}{c_{x}^{-}}+c-v=\frac{c^{2}}{c_{x}^{+}}-c-v \\
\frac{c^{2}}{c_{x}^{-}}=\frac{c^{2}}{c_{x}^{+}}-2 c=\frac{c^{2}-2 c c_{x}^{+}}{c_{x}^{+}}
\end{gathered}
$$

Ultimately, the relation between one-way speed of light in a vacuum parallel to $x^{\prime}$ axis is obtained

$$
c_{x}^{-}=\frac{c c_{x}^{+}}{c-2 c_{x}^{+}}
$$

From formulas (61) and (62) there is a valid conclusion on the parameter $e(v)$. Let us check for what values of the parameter $e(v)$ it occurs, according to what was assumed in relation (2), i.e.

$$
c_{x}^{+} \geq 0 \wedge c_{x}^{-} \leq 0
$$

From (61) and (62) results that there must occur

$$
\left\{\begin{array}{l}
c_{x}^{+}(v) \geq 0 \Rightarrow \frac{\psi(v)}{\gamma(v)} e(v) v c^{2}+c+v \geq 0 \\
c_{x}^{-}(v) \leq 0 \Rightarrow \frac{\psi(v)}{\gamma(v)} e(v) v c^{2}-c+v \leq 0
\end{array}\right.
$$
conditions

It follows that for inequalities (69) to occur, the parameter $e(v)$ must meet the following

$$
\begin{aligned}
& \left\{\begin{array}{l}
c_{x}^{+}(v) \geq 0 \Rightarrow e(v) v \geq \frac{-1}{\psi(v) c} \sqrt{\frac{c+v}{c-v}}=[e(v) v]_{\min } \\
c_{x}^{-}(v) \leq 0 \Rightarrow e(v) v \leq \frac{1}{\psi(v) c} \sqrt{\frac{c-v}{c+v}}=[e(v) v]_{\max }
\end{array}\right. \\
& \begin{array}{l}
1 \\
0.8
\end{array} \\
& 0.6 \\
& 0.4 \\
& 0.2 \\
& 0
\end{aligned}
$$

Fig. 4. Range of function values $e(v) \cdot v$ for which there is no apparent backward travel of light in time.

If conditions (71) are not met for some inertial system then the value of one-way speed of light measured in this system is inconsistent with the time arrow. This means that the light may 
Derivation of all linear transformations that meet the results of Michelson-Morley's experiment and discussion of the relativity basics - Szostek Roman

seem to move back in time (its tide time is negative). This causes the velocity of light $c_{x}^{+}(v)$ that is consistent with $x$-axis direction to be negative, or the velocity of light $c_{x}^{-}(v)$ that is not consistent with $x$-axis direction to be positive. Then one of the inequalities will not be met (69). However, equation (2) is still true, as it is more general than the one adopted at the beginning, for the purpose of determining attention of inequalities (69).

Diagram 4 illustrates the inequalities (71). Functions $[e(v) \cdot v]_{\min }$ and $[e(v) \cdot v]_{\max }$ determine the strip in which the function values $e(v) \cdot v$ must be located in order to prevent any inertial system from experiencing the effect of apparent backward travel of light in time. This diagram also shows the function values $e(v) \cdot v$ for STR (equation (75)) and STE (equation (84)).
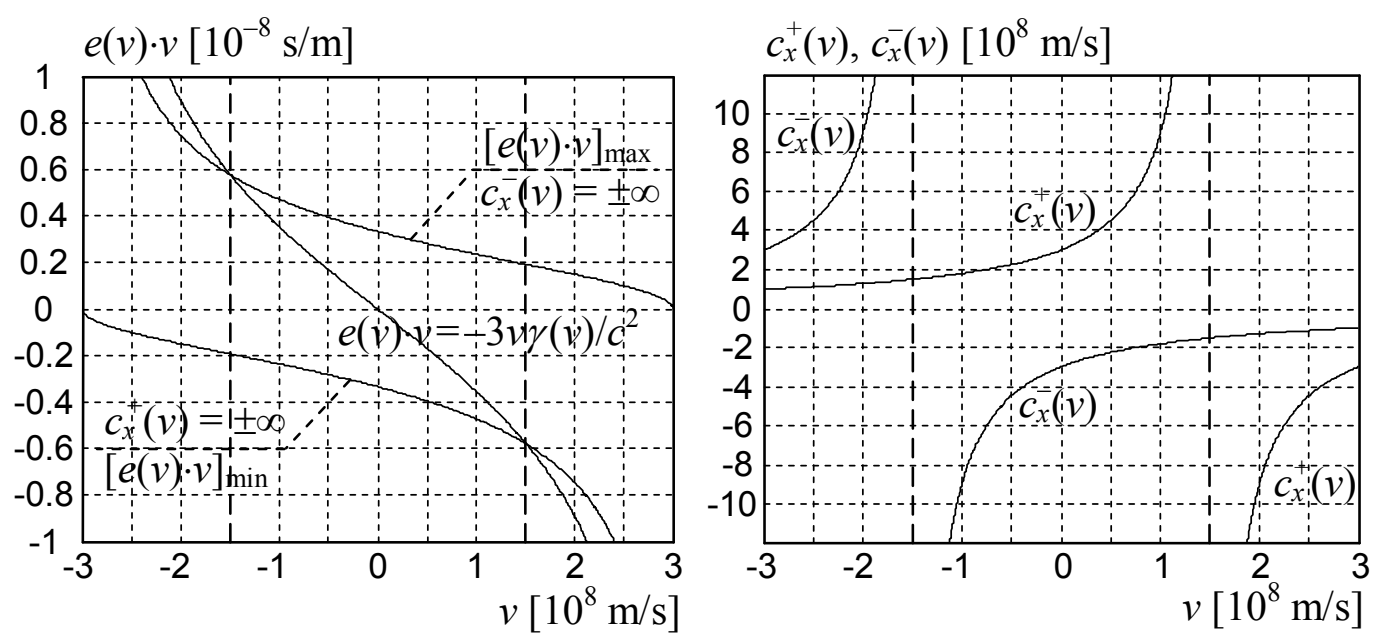

Fig. 5. One-way speed of light in vacuum as a function of velocity $v$, for $e(v) \cdot v=-3 v \gamma(v) / c^{2}$.

Figure 5 shows an example of how the values of one-way speed of light $c_{x}^{+}(v)$ and $c_{x}^{-}(v)$ depend on the speed of inertial system in which the observer is located when the parameter $e(v) \cdot v=-3 v \gamma(v) / c^{2}$.

It is advisable to consider a light impulse moving in the direction of $x$-axis. The impulse moves from point $x_{1}$ to point $x_{2}>x_{1}$. Two clocks are required to measure one-way speed of light $c_{x}^{+}(v)$. The clock $Z_{1}$ at point $x_{1}$ measures the moment $t_{1}$ when a light impulse is emitted. The clock $Z_{2}$ at point $x_{2}$ measures the moment $t_{2}$ when a light impulse reaches it. In case shown in Figure 5, the clocks in inertial systems are so desynchronized with parameter $e(v)$ that in the inertial system moving with velocity $v=c / 2$ there is an equation $t_{1}=t_{2}$. In this situation, the one-way speed of light measurement gives an infinite value as

$$
c_{x}^{+}(c / 2)=\frac{x_{2}-x_{1}}{t_{2}-t_{1}}=\frac{L}{ \pm 0}= \pm \infty
$$

In fact, light in this inertial system has finite velocity because it has finite velocity in the ether. The infinite velocity in (72) is caused by desynchronization of clocks. The clock $Z_{2}$ is exactly as late in comparison to the clock $Z_{1}$ as the time it takes for the light to reach from point $x_{1}$ to point $x_{2}$. Thus, equations (61) and (62) do not represent the actual velocity of light in a vacuum, but the measurement result of this velocity using clocks that are desynchronized when $e(v) \neq 0$.

In the inertial systems (Figure 5) moving at velocities $v>c / 2$ the clocks are already so desynchronized that the measurement of one-way speed of light $c_{x}^{+}(v)$ gives negative values. This is due to the fact that clock $Z_{2}$ is late in comparison with clock $Z_{1}$ for more time than the light needs to reach from point $x_{1}$ to point $x_{2}$. In these inertial systems $t_{2}-t_{1}<0$. Therefore, the light moving from point $x_{1}$ to point $x_{2}$ apparently reverses in time. 
Derivation of all linear transformations that meet the results of Michelson-Morley's experiment and discussion of the relativity basics - Szostek Roman

The fact that the moving back light in time is apparent, not real, will also be explained in subsection 7.7. The significance of parameter $e(v)$ is also discussed in Chapters 7.7, 8 and Bląd! Nie można odnaleźć źródła odwołania..

\section{General form of transformations expressed from one-way speed of light}

In transformations (46)-(47), it is possible to replace the parameter $e(v)$ with one-way speed of light $c_{x}^{+}(v)$ thanks to relation (63). Then the transformation from universal frame of reference $U$ to inertial frame of reference $U^{\prime}$ is obtained in the form of

$$
\left\{\begin{array}{l}
t^{\prime}=\frac{\gamma}{\psi} \cdot \frac{c^{2}-c_{x}^{+}(c+v)}{c^{2} c_{x}^{+}} x+\left(\frac{1}{\gamma \psi}-\frac{\gamma}{\psi} \cdot \frac{c^{2}-c_{x}^{+}(c+v)}{c^{2} c_{x}^{+}} v\right) t \\
x^{\prime}=\frac{\gamma}{\psi}(x-v t) \\
y^{\prime}=\frac{1}{\psi} y \\
z^{\prime}=\frac{1}{\psi} z
\end{array}\right.
$$

However, from the inertial frame of reference $U^{\prime}$ to the universal frame of reference $U$, the transformation takes the form of

$$
\left\{\begin{array}{l}
t=-\gamma \psi \frac{c^{2}-c_{x}^{+}(c+v)}{c^{2} c_{x}^{+}} x^{\prime}+\gamma \psi t^{\prime} \\
x=\left(\frac{\psi}{\gamma}-\gamma \psi \frac{c^{2}-c_{x}^{+}(c+v)}{c^{2} c_{x}^{+}} v\right) x^{\prime}+\gamma \psi v t^{\prime} \\
y=\psi y^{\prime} \\
z=\psi z^{\prime}
\end{array}\right.
$$

In the same way, transformations (46)-(47) can be written on the basis of (64) using oneway speed of light $c_{x}^{-}(v)$.

Thanks to transformations (73)-(74), it is possible to define any transformation that meets the I-IV assumption on the basis of two parameters, i.e. transverse contraction $\psi(v)$ and one-way speed of light in vacuum $c_{x}^{+}(v)$.

Thanks to transformations (46)-(47), it is possible to define any transformation that meets the I-IV assumption on the basis of two parameters, i.e. transverse contraction $\psi(v)$ and the parameter of clock synchronization in inertial frames of reference $e(v)$.

Thanks to transformations (43)-(44), it is possible to define any transformation that meets the I-IV assumption on the basis of two parameters, i.e. time dilation $f(v)$ (resulting from(50)) and the parameter of clock synchronization in inertial frames of reference $e(v)$.

The importance of parameter $e(v)$ is explained further in the article. 
Derivation of all linear transformations that meet the results of Michelson-Morley's experiment and discussion of the relativity basics - Szostek Roman

\section{Special cases of transformation}

\subsection{Lorentz transformation - Special Theory of Relativity transformation}

If we assume that

$$
\begin{aligned}
& \psi(v)=1 \\
& e(v)=-\gamma(v) \frac{1}{c^{2}}=-\frac{1}{\sqrt{1-(v / c)^{2}}} \frac{1}{c^{2}}\left[\frac{\mathrm{s}^{2}}{\mathrm{~m}^{2}}\right]
\end{aligned}
$$

then transformations (46)-(47) take the form of Lorentz transformation on which Special Theory of Relativity is based. From the universal frame of reference $U$ to the inertial frame of reference $U^{\prime}$, the transformation takes the form of

$$
\begin{cases}t^{\prime}=\gamma\left(-\frac{v}{c^{2}} x+t\right) & y^{\prime}=y \\ x^{\prime}=\gamma(x-v t) & z^{\prime}=z\end{cases}
$$

From the inertial frame of reference $U^{\prime}$ to universal frame of reference $U$, the transformation has the following form

$$
\begin{cases}t=\gamma\left(\frac{v}{c^{2}} x^{\prime}+t^{\prime}\right) & y=y^{\prime} \\ x=\gamma\left(x^{\prime}+v t^{\prime}\right) & z=z^{\prime}\end{cases}
$$

There are only two transformations (46)-(47), in which the corresponding coefficients in transformation and in reverse transformation have the same value (with the accuracy to sign resulting from the direction of velocities $v$ ). These are the Lorentz transformation and further shown the Galilean transformation. For this reason, in Lorentz's transformation the systems $U$ and $U^{\prime}$ become indistinguishable.

\subsection{Lorentz transformations with transverse contraction}

If we assume that

$$
e(v)=-\frac{\gamma(v)}{\psi(v)} \frac{1}{c^{2}}=-\frac{1}{\psi(v) \sqrt{1-(v / c)^{2}}} \frac{1}{c^{2}}\left[\frac{\mathrm{s}^{2}}{\mathrm{~m}^{2}}\right]
$$

then transformations (46)-(47) take the form of transformation, which can be called Lorentz transformations with transverse contraction. From the universal frame of reference $U$ to the inertial frame of reference $U^{\prime}$, the transformations take the form of

$$
\begin{cases}t^{\prime}=\frac{\gamma}{\psi}\left(-\frac{v}{c^{2}} x+t\right) & y^{\prime}=\frac{1}{\psi} y \\ x^{\prime}=\frac{\gamma}{\psi}(x-v t) & z^{\prime}=\frac{1}{\psi} z\end{cases}
$$

From the inertial frame of reference $U^{\prime}$ to universal frame of reference $U$, the transformations have the following form 
Derivation of all linear transformations that meet the results of Michelson-Morley's experiment and discussion of the relativity basics - Szostek Roman

$$
\begin{cases}t=\gamma \psi\left(\frac{v}{c^{2}} x^{\prime}+t^{\prime}\right) & y=\psi y^{\prime} \\ x=\gamma \psi\left(x^{\prime}+v t^{\prime}\right) & z=\psi z^{\prime}\end{cases}
$$

It should be explained where the idea of such a generalization of Lorentz's transformation came from in this article. In relation to formula (61) the one-way speed of light raises a natural question, for which parameters $e(v)$ and $\psi(v)$ velocity of light in the inertial frame of reference will have a value $c$ in each direction. On the basis of (61), for light moving along the axis $x^{\prime}$, the equation must be met

$$
\begin{gathered}
c=\frac{c^{2}}{\frac{\psi}{\gamma} e v c^{2}+c+v} \\
\frac{\psi}{\gamma} e v c^{3}+c^{2}+v c=c^{2}
\end{gathered}
$$

I.e. for one-way speed of light to have the exact value $c$, the following must occur

$$
e=-\frac{\gamma}{\psi} \frac{1}{c^{2}}
$$

It is easy to check on the basis of velocity transformations (55) that for all considered transformations (46)-(47) the light moving parallel to $y^{\prime}$ axis will also have value $c$ of one-way speed in $U^{\prime}$ system. It also results directly from assumption IV, which is shown in Figure 2. Also using numerical methods, it was verified for various cases of the function $\psi(v)$ that one-way speed of light in kinematics described by transformations (79)-(80) always has the value $c$.

It follows that there are infinitely many kinematics, in which one-way speed of light in a vacuum, in every inertial frame of reference, is constant and is $c$. They are based on transformations (79)-(80). The Special Theory of Relativity is only one of the infinite number of such kinematics (transformation (76)-(77)).

In kinematics (79)-(80), in which we assume $\psi(v) \neq 1$, the inertial frames of reference are distinguishable, and there is a universal frame of reference, which was indicated by the symbol $U$. The system $U$ is distinguished by the way in which, according to transformation (46)-(47), the lateral dimensions of bodies moving in relation to this system change. Therefore, such theories do not meet the principle of equivalence of all inertial frames of reference.

In modern physics it is believed that one-way speed of light in a vacuum is absolutely constant, i.e. it has the same value in every direction of propagation and for every observer. On this basis STR Einstein was derived. It has been shown above that there are infinitely many kinematics that meet this condition. The STR is distinguished as it additionally assumes the principle of equivalence of all inertial frames of reference, i.e. that there is no such a physical phenomenon, which distinguishes some inertial frame of reference. This comes down to the fact that the corresponding coefficients in transformation and in reverse transformation must have the same value (with the accuracy to sign resulting from the direction of velocities $v$ ). Among transformations (79)-(80), only the Lorentz transformation (76)-(77) meets such an additional assumption. However, there are no experimental grounds for assuming the principle of equivalence of all inertial frames of reference. This principle has been introduced into physics in an arbitrary manner.

Experimental evidence of the existence of a universal frame of reference is known. It is the measurement of anisotropy of cosmic microwave background discussed in the Nobel dissertation [7]. It turns out that electromagnetic cosmic microwave in a range of $300 \mathrm{GHz}$ reaches from all 
Derivation of all linear transformations that meet the results of Michelson-Morley's experiment and discussion of the relativity basics - Szostek Roman

cosmic sides. Cosmic microwave in our frame of reference has dipole anisotropy. Cosmic microwave reaching from the side of Lion constellation has a little more energy, while the one reaching from the side of Aquarius constellation has a little less energy (Figure 6). If Doppler effect is taken into account, it is possible to determine the frame of reference, in which cosmic microwave background is homogeneous. Such a frame of reference is unique in relation to all others. The existence of such a universal frame of reference suggests that even if one-way speed of light in a vacuum was constant, the correct kinematics model is not the Special Theory of Relativity based on Lorentz transformation (76)-(77), but a model based on some other transformation of (79)-(80) form.

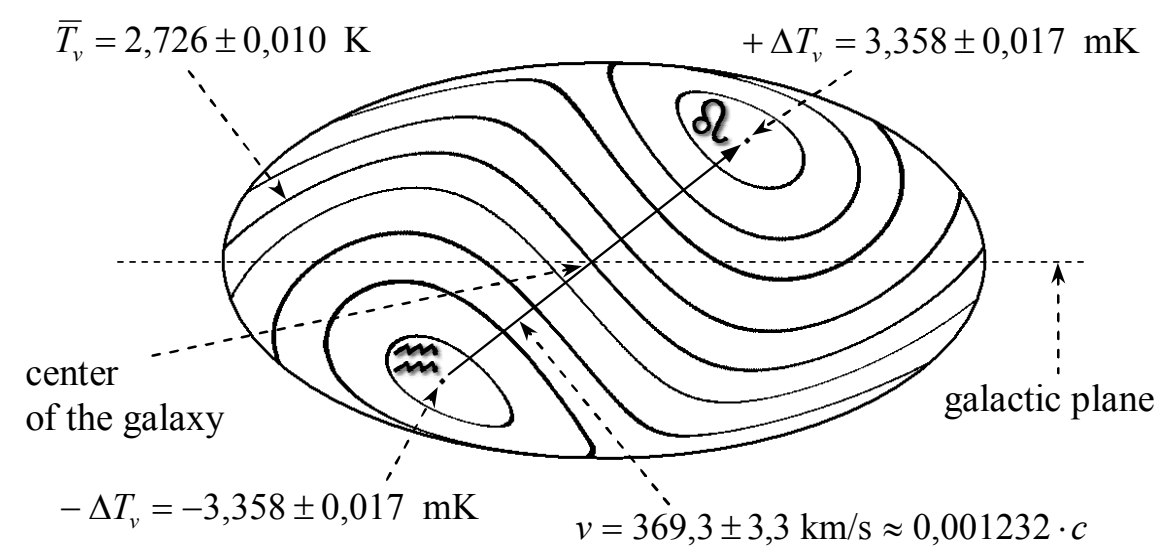

Fig. 6. Dipole anisotropy of cosmic microwave background shown in Hammer-Aitoff projection (own elaboration based on [7]).

In the article [10] on the basis of Special Theory of Ether without transverse contraction, the velocity of Solar System in relation to the system in which cosmic microwave background is homogeneous is determined. A velocity of $369,3 \mathrm{~km} / \mathrm{s}$ was obtained there (Figure 6), but the value of this velocities will be different within other kinematics.

\subsection{Transformations of Special Theory of Ether with transverse contraction}

If we assume that

$$
e(v)=0
$$

then transformations (46)-(47) take the form of transformations on which the Special Theory of Ether is based with transverse contraction derived in the article [10]. From the universal frame of reference $U$ to the inertial frame of reference $U^{\prime}$, the transformations take the form of

$$
\begin{cases}t^{\prime}=\frac{1}{\gamma \psi} t & y^{\prime}=\frac{1}{\psi} y \\ x^{\prime}=\frac{\gamma}{\psi}(x-v t) & z^{\prime}=\frac{1}{\psi} z\end{cases}
$$

From the inertial frame of reference $U^{\prime}$ to universal frame of reference $U$, the transformations have the following form

$$
\begin{cases}t=\gamma \psi t^{\prime} & y=\psi y^{\prime} \\ x=\frac{\psi}{\gamma} x^{\prime}+\gamma \psi v t^{\prime} & z=\psi z^{\prime}\end{cases}
$$


Derivation of all linear transformations that meet the results of Michelson-Morley's experiment and discussion of the relativity basics - Szostek Roman

This is a whole class of transformations in which the simultaneity of events is absolute, which means that the indications of clock do not depend on the coordinates of position. Kinematics based on these transformations differ in physical properties, e.g. transverse contraction and time dilation.

\subsection{Transformation of Special Theory of Ether without transverse contraction}

If we assume that

$$
\begin{aligned}
& \psi(v)=1 \\
& e(v)=0
\end{aligned}
$$

then transformations (46)-(47) take the form of transformation on which the Special Theory of Ether is based without transverse contraction derived in the article [9]. From the universal frame of reference $U$ to the inertial frame of reference $U^{\prime}$, the transformation takes the form of

$$
\begin{cases}t^{\prime}=\frac{1}{\gamma} t & y^{\prime}=y \\ x^{\prime}=\gamma(x-v t) & z^{\prime}=z\end{cases}
$$

From the inertial frame of reference $U^{\prime}$ to universal frame of reference $U$, the transformation has the following form

$$
\begin{cases}t=\gamma t^{\prime} & y=y^{\prime} \\ x=\frac{1}{\gamma} x^{\prime}+\gamma v t^{\prime} & z=z^{\prime}\end{cases}
$$

In this case of the Special Theory of Ether, transverse contraction does not occur (czyli $\psi(v)=1)$. The Special Theory of Ether derived based on transformation (88)-(89) is closely linked to the Special Theory of Relativity by Einstein. This was proven in the work [8].

The transformation (89) was already derived in articles [2], [4] by another method. In those articles the authors obtained such transformation from the Lorentz transformation thanks to the synchronization of clocks in inertial frames of reference by the external method. The transformation obtained in the works [2], [4] is the Lorentz transformation differently written down after a change in the manner of time measurement in the inertial frame of reference, this is why the properties of the Special Theory of Relativity were attributed to this transformation. In the article [9] the transformation (88)-(89) has a different physical meaning than the Lorentz transformation, because according to the theory outlined in that article, it is possible to determine the speed with respect to a universal frame of reference by measurement. So the universal frame of reference is real, and this is not a freely chosen inertial frame of reference.

\subsection{Transformation of Special Theory of Ether with absolute time}

If we assume that

$$
\begin{aligned}
& \psi(v)=1 / \gamma(v)=\sqrt{1-(v / c)^{2}} \leq 1 \\
& e(v)=0
\end{aligned}
$$


Derivation of all linear transformations that meet the results of Michelson-Morley's experiment and discussion of the relativity basics - Szostek Roman

then transformations (46)-(47) take the form of transformation in which the absolute time occurs. From the universal frame of reference $U$ to the inertial frame of reference $U^{\prime}$, the transformation takes the form of

$$
\begin{cases}t^{\prime}=t & y^{\prime}=\gamma y \\ x^{\prime}=\gamma^{2}(x-v t) & z^{\prime}=\gamma z\end{cases}
$$

From the inertial frame of reference $U^{\prime}$ to universal frame of reference $U$, the transformation has the following form

$$
\begin{cases}t=t^{\prime} & y=\frac{1}{\gamma} y^{\prime} \\ x=\frac{1}{\gamma^{2}} x^{\prime}+v t^{\prime} & z=\frac{1}{\gamma} z^{\prime}\end{cases}
$$

In kinematics based on this transformation, time elapses the same way in all inertial frames of reference, just like in Galilean transformations. It is very interesting that the theory with the absolute time which meets the conditions of Michelson-Morley and Kennedy-Thorndike experiments is possible.

\subsection{Transformation of Special Theory of Ether without longitudinal contraction}

If we assume that

$$
\begin{aligned}
& \psi(v)=\gamma(v)=1 / \sqrt{1-(v / c)^{2}} \geq 1 \\
& e(v)=0
\end{aligned}
$$

then transformations (46)-(47) take the form of transformation on which the Special Theory of Ether is based without longitudinal contraction. From the universal frame of reference $U$ to the inertial frame of reference $U^{\prime}$, the transformation takes the form of

$$
\begin{cases}t^{\prime}=\frac{1}{\gamma^{2}} t & y^{\prime}=\frac{1}{\gamma} y \\ x^{\prime}=x-v t & z^{\prime}=\frac{1}{\gamma} z\end{cases}
$$

From the inertial frame of reference $U^{\prime}$ to universal frame of reference $U$, the transformation has the following form

$$
\begin{cases}t=\gamma^{2} t^{\prime} & y=\gamma y^{\prime} \\ x=x^{\prime}+\gamma^{2} v t^{\prime} & z=\gamma z^{\prime}\end{cases}
$$

In kinematics based on this transformation the longitudinal dimensions (parallel to $x$ and $x^{\prime}$ axes) are the same for observers from every inertial frame of reference. This is due to differentials from transformation (94)-(95)

$$
\begin{aligned}
& d x^{\prime}=d x-v d t \\
& d x=d x^{\prime}+\gamma^{2} v d t^{\prime}
\end{aligned}
$$

I.e. 
Derivation of all linear transformations that meet the results of Michelson-Morley's experiment and discussion of the relativity basics - Szostek Roman

$$
\begin{gathered}
d t=0 \quad \Rightarrow \quad d x^{\prime}=d x \\
d t^{\prime}=0 \quad \Rightarrow \quad d x=d x^{\prime}
\end{gathered}
$$

\subsection{Extended Galilean transformations and conclusions on the parameter $e(v)$}

If we assume that

$$
\begin{aligned}
& v / c \rightarrow 0 \Rightarrow \gamma=1 / \sqrt{1-(v / c)^{2}} \cong 1 \\
& \psi(v)=1 \quad \vee \quad(v / c \rightarrow 0 \Rightarrow \psi(v) \cong 1)
\end{aligned}
$$

then transformations (46)-(47) take the forms that can be called the extended Galilean transformations. From the universal frame of reference $U$ to the inertial frame of reference $U^{\prime}$, the transformations take the form of

$$
\begin{cases}t^{\prime}=e v x+\left(1-e v^{2}\right) t & y^{\prime}=y \\ x^{\prime}=x-v t & z^{\prime}=z\end{cases}
$$

From the inertial frame of reference $U^{\prime}$ to universal frame of reference $U$, the transformations have the following form

$$
\begin{cases}t=-e v x^{\prime}+t^{\prime} & y=y^{\prime} \\ x=\left(1-e v^{2}\right) x^{\prime}+v t^{\prime} & z=z^{\prime}\end{cases}
$$

The transformations (99)-(100) applies when velocity $v$ is very small in relation to $c$, or simply when $c=\infty$. Then on the basis of (61) and (62) the one-way speed of light flowing along $x$ axis is

$$
\begin{aligned}
& c_{x}^{+}(v)=c_{x}^{-}(v)=\frac{1}{e(v) v} \\
& c=\infty
\end{aligned}
$$

On the basis of dependency (101), important conclusions can be drawn. One-way speed of light in a vacuum has an infinite value $(c=\infty)$ in relation to the universal frame of reference. Then, of course, this velocity has an infinite value in every inertial system. However, according to (101) the one-way speed of light in the inertial system is $1 /(e v)$. This seems to be contradictory.

a)

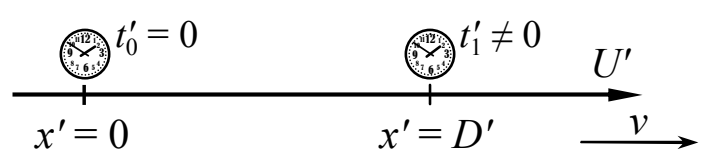

b) $\underset{x=0}{\stackrel{c=-\infty}{\rightleftarrows}} \underset{x=D}{\stackrel{t_{0}=\infty}{t_{0}=0}} \overbrace{}^{t_{1}=0} U$-ether

Fig. 7. Measurement of the velocity of light in an inertial system when the clocks are desynchronized in the system and when the light in the universal frame of reference (ether) has infinite velocity.

However, there is no contradiction in this. It should be noted that in order to measure oneway speed it is necessary to use at least two motionless clocks in the system in which the measurement is carried out. Different items of clocks are used in the universal frame of reference than in the inertial system. If in the inertial system the clocks are desynchronized, the measurement 
Derivation of all linear transformations that meet the results of Michelson-Morley's experiment and discussion of the relativity basics - Szostek Roman

of light speed moving with infinite velocity can give a seeming finite value. This has been illustrated in Figure 7.

In part $b$ ) of the figure, the universal frame of reference is shown. The light is sent from point $x=0$ at the moment $t_{0}$ and immediately reaches point $x=D$ at the moment $t_{1}$. As it is assumed in the universal frame of reference, the velocity of light is infinite, $t_{0}=t_{1}=0$.

In part $a$ ) of the figure, the inertial system $U^{\prime}$ is shown. At the moment under consideration, the two clocks of this system are located directly next to the two clocks of system $U$. Due to length contraction, the distance of clocks measured in system $U^{\prime}$ is $D^{\prime}$ and may have a different value than $D$ measured in system $U$. The clock at point $x^{\prime}=0$ indicates the time $t_{0}^{\prime}=0$, but the clock at point $x^{\prime}=D^{\prime}$ indicates another time $t_{1}^{\prime} \neq 0$. According to the observer from system $U^{\prime}$, the light was sent at the moment $t_{0}^{\prime}=0$, while to point $D^{\prime}$ it reached at the moment $t_{1}^{\prime} \neq 0$. Therefore, in system $U^{\prime}$ the one-way speed of light is

$$
c_{x}^{+}(v)=\frac{D^{\prime}}{t_{1}^{\prime}-t_{0}^{\prime}} \quad\left\{\begin{array}{l}
<\infty \\
>-\infty
\end{array}\right.
$$

The velocity (102) is the result of a measurement carried out by desynchronized clocks in system $U^{\prime}$. It is not a true velocity of light, which in this case has an infinite value. What's more, the clocks in the inertial system $U^{\prime}$ can be so desynchronized that the light will move back in time. It will be so when $t_{1}^{\prime}<t_{0}^{\prime}$. Such cases were discussed in Chapter 5.5.

A similar situation is obtained when the light moves in the opposite direction, i.e. from point $x=D$ to point $x=0$.

The situation shown in Figure 7 occurs for transformations where the parameter $e(v) \neq 0$ (e.g. in the Special Theory of Relativity). Then the clocks passing next to each other already at the moment of their synchronization indicate other values. The above example shows that then the oneway speed of light (61) and (62) measured in inertial systems do not reflect the real velocity of light in these systems.

Therefore, the parameter $e(v) \neq 0$ causes desynchronization of clocks. Readings from such clocks should not be taken literally, and theories based on such a parameter, such as STR, should be interpreted differently than it is done in modern physics. This subject will be further elaborated in Chapter 8.

In case of the extended Galilean transformations in the inertial frame of reference $U^{\prime}$ (but not in system $U$ ), the clock indications have been rearranged (the clocks have been desynchronized) to the natural setting occurring in Galilean transformation. For this reason, in time transformations there is a factor depending on the position $x$ or $x^{\prime}$.

\subsection{Galilean transformation}

If we assume that

$$
\begin{aligned}
& v / c \rightarrow 0 \Rightarrow \quad \gamma=1 / \sqrt{1-(v / c)^{2}} \cong 1 \\
& \psi(v)=1 \quad \vee \quad(v / c \rightarrow 0 \Rightarrow \psi(v) \cong 1) \\
& e(v)=0 \quad \vee \quad(v / c \rightarrow 0 \Rightarrow \quad e(v) \cong 0)
\end{aligned}
$$

then transformations (46)-(47) take the form of Galilean transformation on which classical kinematics is based. From the universal frame of reference $U$ to the inertial frame of reference $U^{\prime}$, the transformation takes the form of 
Derivation of all linear transformations that meet the results of Michelson-Morley's experiment and discussion of the relativity basics - Szostek Roman

$$
\begin{cases}t^{\prime}=t & y^{\prime}=y \\ x^{\prime}=x-v t & z^{\prime}=z\end{cases}
$$

From the inertial frame of reference $U^{\prime}$ to universal frame of reference $U$, the transformation has the following form

$$
\begin{cases}t=t^{\prime} & y=y^{\prime} \\ x=x^{\prime}+v t^{\prime} & z=z^{\prime}\end{cases}
$$

In Galilean transformation, the corresponding coefficients in transformation and in reverse transformation have the same value (with accuracy to the sign resulting from the direction of velocities $v$ ). For this reason, in the Galilean transformation, as in the Lorentz transformation, the $U$ and $U^{\prime}$ systems become indistinguishable.

The Galilean transformation can be treated as an approximation of all linear transformations derived in this article for small velocities $v$, i.e. when $v<<c$. Therefore, classical kinematics is consistent with experiments on small velocities $v$ regardless of which of the infinitely many possible kinematics is the best model of real processes.

The transformation (104)-(105) applies when velocity $v$ is very small in relation to $c$, or simply when $c=\infty$. Then on the basis of (61) and (62) the one-way speed of light flowing along $x$ axis is

$$
c_{x}^{+}(v)=-c_{x}^{-}(v)=c=\infty
$$

\section{Physical significance of parameters occurring in transformations and discussion of relativity basics}

\subsection{Parameters $f(v), a(v)$ and $\psi(v)$}

From formulas (50) and (51) for time dilation, it results that the parameter $f(v)$, which occurs in transformation (43)-(44), describes time dilation. For the motionless observer in relation to universal frame of reference, the time in inertial frame of reference elapses $f(v)$ times faster $(1 / f(v)$ times slower) than in its universal frame of reference.

From formulas (52) and (53) for longitudinal contraction it results that parameter $a(v)$, which occurs in transformations (5) and (11), describes longitudinal contraction (i.e. parallel to velocities $v$ ) of bodies in motion in relation to bodies resting in relation to the universal frame of reference $U$. For the motionless observer in relation to universal frame of reference, the moving body is $a(v)$ times shorter (1/a(v) times longer) than the same motionless body in relation to the universal frame of reference.

The parameter $\psi(v)$, which occurs in transformation (46)-(47), describes transverse contraction (perpendicular to velocities $v$ ) of bodies moving in relation to bodies resting in relation to the universal frame of reference $U$ (Figure 2). That is, the moving body is $\psi(v)$ times wider $(1 / \psi(v)$ times narrower) than the same motionless body in relation to the universal frame of reference. 
Derivation of all linear transformations that meet the results of Michelson-Morley's experiment and discussion of the relativity basics - Szostek Roman

\subsection{Parameter $e(v)=0$}

On the basis of transformations (43)-(44) and (46)-(47) it can be concluded that parameter $e(v)$ can be treated as a way of clock synchronization in the inertial frames of reference. Consider the case when $e(v)=0$. Then the time transformation (85), from which the following is obtained

$$
t^{\prime}=\frac{1}{\gamma \psi} t \Rightarrow\left(t=0 \Rightarrow t^{\prime}=0\right)
$$

This means that for $e(v)=0$, the clock synchronization in system $U^{\prime}$ consists in the fact that if the clock of system $U$ indicates time $t=0$, then according to (107), the clock of system $U^{\prime}$ next to it is also reset to zero, that is $t^{\prime}=0$. This way of synchronization is shown in Figure 8.

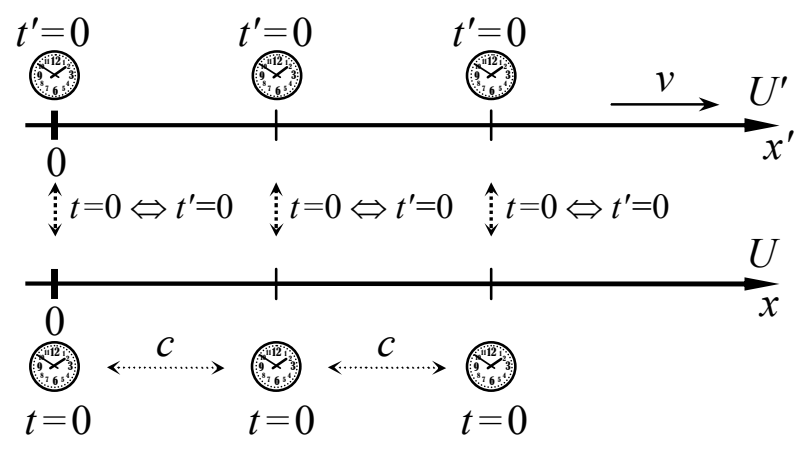

Fig. 8. External clock synchronization $(e(v)=0)$.

The clocks in universal frame of reference $U$ were synchronized with light, which in this system has one-way speed $c$. In the time under consideration, when all clocks of the system $U$ indicate time $t=0$, the beginnings of systems $U$ and $U^{\prime}$ coincide. On each clock passing by clock $t=0$ the time is also set $t^{\prime}=0$.

In this particular case, when $e(v)=0$, one-way speed of light (61) and (62) take the following values

$$
\begin{gathered}
e(v)=0 \Rightarrow c_{x}^{+}(v)=\frac{c^{2}}{c+v} \\
e(v)=0 \Rightarrow c_{x}^{-}(v)=-\frac{c^{2}}{c-v}
\end{gathered}
$$

\subsection{Parameter $e(v) \neq 0$}

Let's consider cases for any parameter $e(v)$. Transformation of time (47) has the form of

$$
t=-\psi^{2} e v x^{\prime}+\gamma \psi t^{\prime}
$$

When the clocks in system $U$ indicate time $t=0$, then according to Figure 1 the beginnings of systems coincide. From equation (110) it results that on the clock from system $U^{\prime}$ located next to zeroed clock from system $U$, the value $t^{\prime}$ is set, which is expressed by the formula

$$
t=0 \Rightarrow t^{\prime}=\frac{\psi e v}{\gamma} x^{\prime}
$$

Synchronization of clocks in the system $U^{\prime}$ is shown in Figure 9. From the perspective of system $U$, clocks in the system $U^{\prime}$ are desynchronized, as their indications depend on the position and not only on the time lapse. If the observer from system $U^{\prime}$ measures one-way speed of light, 
Derivation of all linear transformations that meet the results of Michelson-Morley's experiment and discussion of the relativity basics - Szostek Roman

then on some clock reads the initial time, while on another clock measures the final time. These clocks can always be set in such a way (desynchronize them) that one-way speed of light will have the predetermined value of $c_{x}^{+}(v)$ and $c_{x}^{-}(v)$. The same effect can also be achieved in classical mechanics. If to move forward the indications of clock from which the final time is read, the seemingly velocity of body will be smaller, while if to move back the indications of this clock, the seemingly velocity of body will be larger.

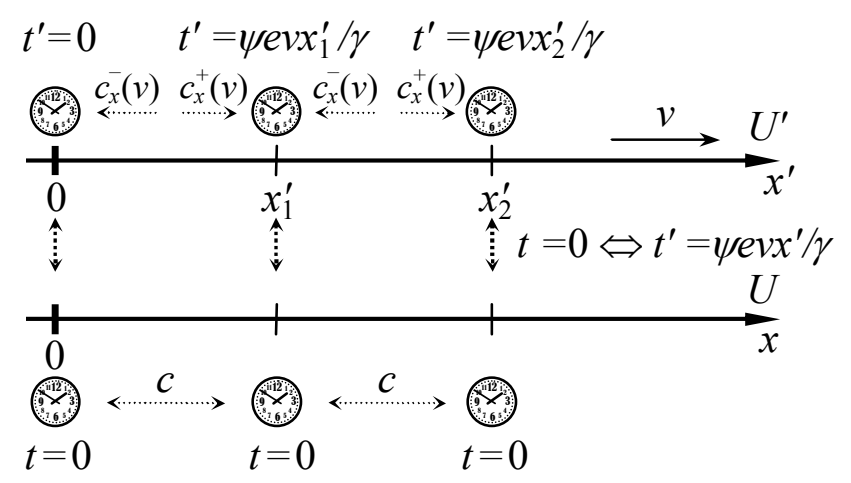

Fig. 9. External clock synchronization $(e(v) \neq 0)$.

The clocks in universal frame of reference $U$ were synchronized with light, which in this system has one-way speed $c$. In the time under consideration, when all clocks of the system $U$ indicate time $t=0$, the beginnings of systems $U$ and $U^{\prime}$ coincide. On each clock passing by clock $t=0$ the set time $t^{\prime}=\psi e v x^{\prime} / \gamma$.

For considered transformations, if the clocks in system $U^{\prime}$ are set according to formula (111) then one-way speed of light will have the value expressed in formulas (17), (18) and (61), (62). However, this does not mean that it is a velocity resulting from the speed at which the real processes on which the clocks are based take place. This can only be the result of setting the clocks in inertial frames of reference as shown in Figure 9. As kinematics model should be expected to describe real processes, clocks in inertial frames of reference cannot be set freely, only in a way that corresponds to the described processes.

If $e(v) \neq 0$, then formulas (50) and (51) are different, as well as formulas (52) and (53) are different. Therefore, observers from $U$ and $U^{\prime}$ systems draw different conclusions on time dilation and longitudinal contraction on the basis of their measurements (they evaluate the relative passage of time in their systems differently and they evaluate the proportions of horizontal lines in their systems differently). Such a situation can be interpreted in such a way that their measuring devices have not been synchronized and for this reason they measure something else. Only if $e(v)=0$, then their measurements of time dilation and longitudinal contraction give the same result, i.e. only then the clocks with their frames of reference were correctly synchronized.

Consider the light impulse sent to the right from the beginning of system $U^{\prime}$ in time synchronization of clocks (Figure 9). In time $t_{1}^{\prime}=0$, the impulse was in position $x_{1}^{\prime}=0$, while in time $t_{2}^{\prime}$ it was in position $x_{2}^{\prime}$. On the basis of (61), it is possible to write that

$$
t_{2}^{\prime}-t_{1}^{\prime}=t_{2}^{\prime}=\frac{x_{2}^{\prime}-x_{1}^{\prime}}{c_{x}^{+}(v)}=\frac{x_{2}^{\prime}}{c_{x}^{+}(v)}=\frac{\frac{\psi}{\gamma} e v c^{2}+c+v}{c^{2}} x_{2}^{\prime}=\frac{\psi e v}{\gamma} x_{2}^{\prime}+\frac{x_{2}^{\prime}}{\frac{c^{2}}{c+v}}
$$

Formula (112) may have different interpretations. In the Special Theory of Relativity, the interpretation is that clocks in the inertial frame of reference $U^{\prime}$ are correctly synchronized. Therefore, the light actually needed $t_{2}^{\prime}$ time to travel the distance of $x_{2}^{\prime}$. Then actually for the observer from system $U^{\prime}$ the light has one-way speed expressed by the formula (61). For STR on the basis of (75) this velocity has the value of 
Derivation of all linear transformations that meet the results of Michelson-Morley's experiment and discussion of the relativity basics - Szostek Roman

$$
c_{x}^{+}(v)=\frac{c^{2}}{\frac{\psi}{\gamma} e v c^{2}+c+v}=\frac{c^{2}}{-\frac{1}{\gamma} \gamma \frac{1}{c^{2}} v c^{2}+c+v}=c
$$

However, it is important to remember that the values $t_{2}^{\prime}$ and $t_{1}^{\prime}=0$ are read from two different clocks. If these clocks are not synchronized correctly, then the velocity (113) is apparent. Then the velocity (61) does not reflect the course of physical processes, but is caused by the way clocks are set in the inertial frame of reference $U^{\prime}$. For such an interpretation, after taking into account (108), the formula (112) will be written in the following form

$$
t_{2}^{\prime}-\frac{\psi e v}{\gamma} x_{2}^{\prime}=\frac{x_{2}^{\prime}}{\frac{c^{2}}{c+v}}=\frac{x_{2}^{\prime}}{c_{x}^{+}(e=0)}
$$

That is, when a light impulse was emitted, then the value (111) was set on the clock at point $x_{2}^{\prime}$, but in reality the value of 0 resulting from the correct synchronization, i.e. from formula (107), should be set. Therefore, when the impulse reaches point $x_{2}^{\prime}$, then the correct indication of a clock in that point is not $t_{2}^{\prime}$, but only

$$
t_{2}^{\prime}-\frac{\psi e v}{\gamma} x_{2}^{\prime}
$$

With this interpretation, the left side of equation (114) is the real time that an impulse needed to reach point $x_{2}^{\prime}$. If the clock at point $x_{2}^{\prime}$ is correctly synchronized according to the formula (107), then the one-way speed of light will be (108) or (109), and not (61) or (62).

It follows from the above that if the parameter $e(v) \neq 0$, then different interpretations of transformation (43)-(44) and (46)-(47) are possible. In the Special Theory of Relativity the interpretation is that readings from the clocks should be treated literally in this situation. This leads to the fact that different observers measuring the same physical phenomena obtain different results (except for one-way speed of light in a vacuum). In STR it was considered to be a property of space-time and not a result of synchronization of clocks between inertial frames of reference.

For the second interpretation of the parameter $e(v)$, adopting that $e(v) \neq 0$ causes the clocks to be desynchronized in the inertial frame of reference, but all the time it is the same kinematics as the one based on parameter $e(v)=0$. After the clocks are desynchronized the values shown by these clocks should not be treated literally. If the calculation takes into account the fact that clocks are desynchronized, then each kinematics with the parameter $e(v) \neq 0$ comes down to kinematics with the parameter $e(v)=0$. According to this interpretation, the parameter $e(v)$ does not allow other kinematics to be obtained. All kinematics possible for the I-IV assumptions adopted in this article are included in transformations (85)-(86). Kinematics differ in only one parameter of transverse contraction $\psi(v)$. In monograph [8] it is shown, that with such an interpretation STR becomes STE with a universal frame of reference. According to this interpretation, numerous conclusions of modern physics drawn from the mathematics on which STR is based are incorrect. Therefore, STR mathematics is correct, but the interpretation of this mathematics is not correct.

Introduction of parameter $e(v) \neq 0$ for the Galilean transformation (104)-(105) leads to transformation (99)-(100). This means that after desynchronization of clocks between different inertial frames of reference, a transformation is obtained in which the values indicated by the clocks of system $U^{\prime}$ depend on their position. However, it is still classical kinematics, only written in a more complex way. After all, the way of setting the initial values on the clocks of system $U^{\prime}$ has no influence on the course of physical processes in classical mechanics. However, it is formally possible to write this kinematics with the parameter $e(v) \neq 0$. If in classical kinematics written with transformation (99)-(100), the values indicated by desynchronized clocks are treated literally, then 
Derivation of all linear transformations that meet the results of Michelson-Morley's experiment and discussion of the relativity basics - Szostek Roman

there are similar conclusions to those drawn from the Lorentz transformation in the Special Theory of Relativity. That is, for example, that the simultaneity of events, time dilation and longitudinal contraction are relative. However, if the second interpretation is adopted, then all kinematics (99)(100) come down to the classical kinematics described by transformations (104)-(105).

In this article, the interpretation of parameter $e(v)$ was adopted in such a way that it describes the desynchronization of clocks in inertial frames of reference. In monograph [8], in chapter "What is the Special Theory of Relativity (STR)" it was shown that the parameter $e(v)$ can be assigned another, third interpretation. The parameter $e(v)$ can describe displacement in time and space that the transformation implements. It is commonly believed that the transformation is related to clocks that are directly adjacent to each other in a given time. That is, it recalculates coordinates of the same event seen from different frames of reference. This is how Lorentz's transformation in the Special Theory of Relativity is understood. But the transformation can calculate the coordinate of clock's position to the coordinate of the same clock in another frame of reference, but one at which the clock will be in the future or was in the past. With this interpretation, the transformation does not calculate the coordinates of the same event, but the coordinates of different events. With this interpretation of parameter $e(v)$ the transformation is related to coordinates of the same event only if $e(v)=0$.

\section{Importance of parameter $e(v)$ for time dilation}

In case of transformations, in which the parameter $e(v) \neq 0$ is not possible to state directly from the clock readings that in some inertial system time elapses slower or faster than in universal frame of reference (or other inertial system).

Let us consider the situation shown in Figure 10. In the inertial system $U^{\prime}$ there is an observer $O^{\prime}$, while in the universal system $U$ there is an observer $O$.

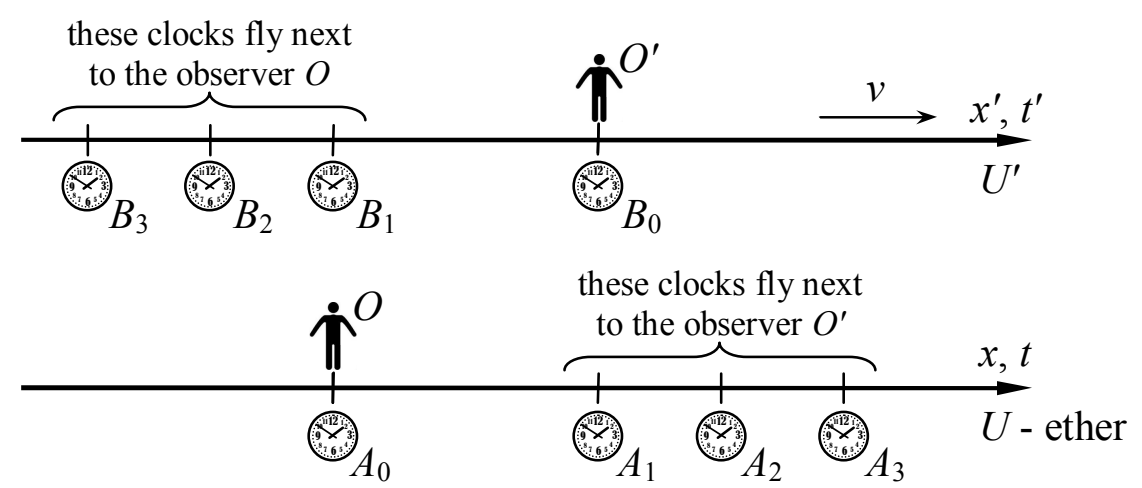

Fig. 10. Times measured by two observers from different reference systems.

The observer $O^{\prime}$ has in his system $B_{i}$ clocks. The clock $B_{0}$ is located directly next to him. The observer $O$ has in his system $A_{i}$ clocks. The clock $A_{0}$ is located directly next to him. Observers $O$ and $O^{\prime}$ cannot directly compare $A_{0}$ and $B_{0}$ clocks because they are far apart and in constant relative motion.

Each of these observers can at any time read the time from the two clocks that are directly next to him. The observer $O$ can read the time from his $A_{0}$ clock and from $B_{i}$ clock passing by. From a particular $B_{i}$ clock he can read the time only once, when that particular clock is next to him. Each time he reads the time from $B_{i}$ clock, it is a different clock. 
Derivation of all linear transformations that meet the results of Michelson-Morley's experiment and discussion of the relativity basics - Szostek Roman

The same applies to the observer $O^{\prime}$. The observer $O^{\prime}$ can read the time from his $B_{0}$ clock and from $A_{i}$ clock passing by. He can read the time from a specific $A_{i}$ clock only once when that particular clock is next to him. Each time he reads the time from $A_{i}$ clock, it is a different clock.

The observer can read the time from the clock that is currently next to him. He can also read the position of such a clock according to the coordinates of $U$ and $U^{\prime}$ systems. The coordinate and time transformation derived in this paper are used to convert readings carried out according to one reference system to readings carried out according to another reference system.

In the situation under consideration, the time dilation equation (50) applies to the observer $O$ from the universal system, while the time dilation equation (51) applies to the observer $O^{\prime}$ from the inertial system. In other words, the observer $O$ evaluates the relative time elapse on the clocks he compares in the following way

$$
d x=0 \Rightarrow \frac{d t^{\prime}}{d t}=\frac{1}{\gamma \psi}-e v^{2}
$$

In turn the observer $O^{\prime}$ evaluates the relative elapse of time on the compared clocks in the following way

$$
d x^{\prime}=0 \Rightarrow \frac{d t^{\prime}}{d t}=\frac{1}{\gamma \psi}
$$

This means that if $e(v) \neq 0$, then the observers $O$ and $O^{\prime}$ will evaluate the relative time elapse differently (this has already been noted in subsection 5.1). It should also be noted that the observer $O$ reads the time from clocks other than the observer $O^{\prime}$.

For the observer $O$ from the universal system, the time elapsed on $B_{i}$ clocks is not measured by one clock, but by many clocks passing one after the other. If $B_{i}$ clocks are desynchronized, then the observer $O$ reading from them does not measure the real time elapse in $U^{\prime}$ system. In equation (116) there is the factor $e(v) \cdot v^{2}$, which describes how the desynchronization of $B_{i}$ clocks affects the time dilation, measured by the observer $O$.

The observer $O^{\prime}$ from the inertial system evaluates time dilation on the basis of $A_{i}$ clocks. These clocks are motionless in relation to the ether and have been desynchronized by means of light, which in assumption has one-way speed in the ether with a constant value of $c$. The dependence (117) shows that parameter $e(v)$ does not affect time dilation measurement, which is performed by the observer $O^{\prime}$. Therefore, $A_{i}$ clocks were not desynchronized with this parameter. The observer $O^{\prime}$ reads the real time elapse in the universal frame of reference $U$.

If $e(v) \neq 0$, then the time dilation measurements by the observer $O$ and $O^{\prime}$ are different. Therefore, it is not objectively possible to state that time elapses faster in one system and slower in another. However, it can be stated that their clocks are not desynchronized.

The fact that parameter $e(v)$ describes how to desynchronize clocks in the inertial systems can also be deduced from the time dilation dependency (50). The observer $O$, shown in Figure 10, is motionless relative to the universal frame of reference $U$ and evaluates the time elapse in its $U$ system on the basis of one $A_{0}$ clock. As all clocks measure time according to the time arrow, the time elapse on $A_{0}$ clock meets the condition $d t>0$. Based on (50) the following is obtained

$$
\left(d x=0 \wedge d t>0 \wedge e(v)>\frac{1}{\gamma(v) \psi(v) v^{2}}\right) \Rightarrow d t^{\prime}<0
$$

In other words, the observer $O$ reads the time on successive $B_{i}$ clocks and states that a later reading indicate an earlier, not later, moment of time. If the observer $O$ treated such readings literally, as is currently done in the Special Theory of Relativity, he would conclude that in the inertial system $U^{\prime}$ time reverses. However, this is not true, because by definition each $B_{i}$ clock 
Derivation of all linear transformations that meet the results of Michelson-Morley's experiment and discussion of the relativity basics - Szostek Roman

measures time according to the time arrow. The reason for such a strange measurement is that the observer $O$ when reading the time on $B_{i}$ clocks does not measure the actual time elapse in the inertial system $U^{\prime}$. Its measurement is influenced by how the clocks of $U^{\prime}$ system are desynchronized by the parameter $e(v)$. That is, if $B_{i+1}$ clock is very late in relation to $B_{i}$ clock $\left(t_{i+1}<<t_{i}\right)$, then the time elapse that the observer $O$ will measure on $B_{i}$ and $B_{i+1}$ clocks will be negative $\left(t_{i+1}-t_{i}<0\right)$. The way the clocks are desynchronized is shown in Figure 9. In order for this effect to occur, the parameter $e(v)$ must has positive and enough large values. This has been shown in Figure 11.

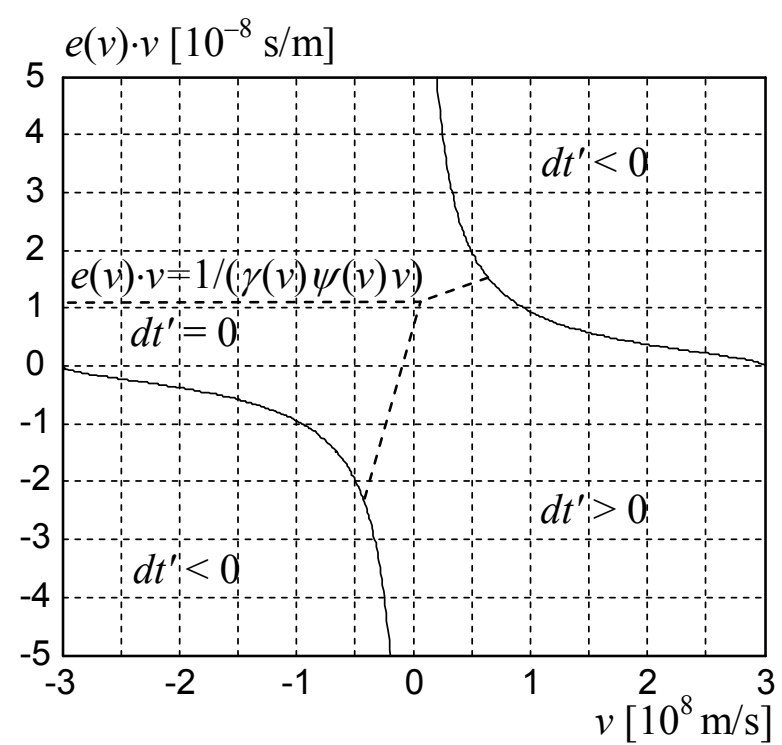

Fig. 11. Influence of parameter $e(v)$ on time measurement in a mobile inertial system by an observer with universal frame of reference $(d t>0)$.

This issue has been addressed in another way in Chapter 7.7, where, on the example of Galilean extended transformations, it has been shown that evaluating the time elapse on the basis of two different clocks, even if they are from the same inertial system, leads to misleading conclusions if the parameter $e(v) \neq 0$. In this case, if the observer reads $t_{0}$ on one clock and $t_{1}$ on another, it does not mean that time $t_{1}-t_{0}$ passed between readings. The difference $t_{1}-t_{0}$ is affected by the actual time elapse, but also by the extent to which the two clocks are desynchronized.

\section{Conclusion}

The article presents the original method of studying the transformations of time and coordinate in terms of acceptable interpretations that can be attributed to these transformations.

All possible linear transformations that meet the results of Michelson-Morley's and Kennedy-Thorndikea's experiments (without turnovers) have been derived in this article. On the basis of these transformations it is possible to build numerous kinematics with different physical properties. Thus, there are infinitely many kinematics consistent with experiments in which velocity of light was measured.

For each kinematics an infinite number of dynamics can be derived. The method that allows this is shown in the papers [8] and [12].

In this article it was also shown that there are infinitely many different kinematics, in which one-way speed of light in a vacuum has in each direction and in each inertial frame of reference the 
Derivation of all linear transformations that meet the results of Michelson-Morley's experiment and discussion of the relativity basics - Szostek Roman

value of $c$ (transformations (79)-(80)). The Special Theory of Relativity is only one of those infinitely many kinematics.

The phenomenon of dipole anisotropy of cosmic microwave background proves that there is a universal frame of reference, in which this cosmic microwave background is homogeneous. This shows that in reality the inertial frames of reference are experimentally distinguishable, i.e. they are not equivalent. It follows that even if we assume that one-way speed of light is absolutely constant, the Special Theory of Relativity is not the correct model of real processes. If one-way speed of light is absolutely constant, then the correct model of real processes will be kinematics based on one of the Lorentz transformation with transverse contraction (79)-(80).

Kinematics, in which one-way speed of light is always constant, are possible if one assumes the interpretation of parameter $e(v)$ as adopted in the Special Theory of Relativity, i.e. that they describe specific properties of space-time and not the desynchronization of clocks in inertial frames of reference.

However, the parameter $e(v)$ can be interpreted differently, i.e. in such a way that it describes the way of desynchronizing clocks in inertial frames of reference in relation to the universal frame of reference. In this article the thesis was formulated that assuming the parameter $e(v) \neq 0$ leads to desynchronization of clocks between different inertial frames of reference. However, the way of setting the initial values on clocks located in inertial frames of reference does not affect the physical processes. If such an interpretation of this parameter is assumed, then any kinematics with parameter $e(v) \neq 0$ comes down to kinematics based on parameter $e(v)=0$. Therefore, the only parameter that can differ from kinematics meeting the I-IV assumptions is the transverse contraction parameter $\psi(v)$. Then it is not possible kinematics in which one-way speed of light is constant in every inertial frame of reference. All kinematics are included in transformations (85)-(86). The one-way speed of light formula for these kinematics has been derived in this article [10].

The paper shows that interpretation of parameter $e(v)$ as assumed in STR leads to contradiction (Chapter 7.7). Therefore, the commonly accepted in physics interpretation of this parameter is mistaken. The correct interpretation of this parameter is that if $e(v) \neq 0$, then the clocks in inertial systems are desynchronized. Then it is not possible to compare readings from different clocks of the same inertial system literally.

Regardless of how the parameter $e(v)$ is interpreted, parameter $\psi(v)$ is not a variation of scale. This is obvious, if it is noticed that in every inertial system the measurements are carried out using technically identical devices. Firstly, in some inertial system the identical clocks for time measurement and identical rulers for distance measurement are generated. Then some of these devices are transferred to other inertial systems. Transformations derived in this article describe the relations between measurements carried out by such identical devices placed in the universal frame of reference and various inertial systems. Any change to parameter $\psi(v)$ changes the physical properties of kinematics. It is sufficient to note that this parameter determines the time dilation described by the formula (51). That is, the way in which time is measured by clocks moving in relation to the universal frame of reference depends on the value of parameter $\psi(v)$.

All experiments conducted by man were observed with inertial frames of reference moving with small velocities relative to universal frame of reference. Such experiments do not provide an answer on how the laws of nature look like for observers found in the inertial frames of reference moving with large velocities relative to universal frame of reference. Therefore, in physical theories, the results obtained in frames of reference available to the observer are extrapolated to all other inertial frames of reference. But as, they are acceptable as valid models of real processes, kinematics based on transformations that do not meet the III assumption in all inertial frames of reference, but only in inertial frames of reference available for experiments. The introduction of such transformations is presented in the article [13]. 
Derivation of all linear transformations that meet the results of Michelson-Morley's experiment and discussion of the relativity basics - Szostek Roman

\section{Bibliography}

[1] Kennedy Roy J., Thorndike Edward M., Experimental Establishment of the Relativity of Time, Physical Review, 42 (3), 400-418, 1932.

[2] Mansouri Reza, Sexl Roman U., A Test Theory of Special Relativity: I. Simultaneity and Clock Synchronization, General Relativity and Gravitation, Vol. 8, No. 7, 497-513, 1977.

[3] Michelson Albert A., Morley Edward W., On the relative motion of the earth and the luminiferous ether, Am. J. Sci. 34, 333-345, 1887.

[4] Rizzi Guido, Ruggiero Matteo L., Serafini Alessio, Synchronization Gauges and the Principles of Special Relativity, Foundations of Physics, Vol. 34, 1835-1887, No. 12, 2004.

[5] Selleri Franco, Noninvariant One-Way Velocity of Light, Foundations of Physics. Vol. 26. No. 5, 1996, 641-664.

[6] Selleri Franco, Noninvariant One-Way Speed of Light and Locally Equivalent Reference Frames, Foundations of Physics Letters, Vol. 10, No. 1, 1997, 73-83.

[7] Smoot George F., Anizotropie kosmicznego mikrofalowego promieniowania tła: ich odkrycie $i$ wykorzystanie (in Polish), Postępy Fizyki, Tom 59, Zeszyt 2, 52-79, 2008.

Smoot George F., Nobel Lecture: Cosmic microwave background radiation anisotropies: Their discovery and utilization (in English), Reviews of Modern Physics, Volume 79, 1349-1379, 2007.

Смут Джордж Ф., Анизотропия реликтового излучения: открытие и научное значение (in Russian), Успехи Физических Наук, Том 177, № 12, 1294-1317, 2007.

[8] Szostek Karol, Szostek Roman, Special Theory of Ether (in English), Publishing house AMELIA, Rzeszów, Poland, 2015, (www.ste.com.pl), ISBN 978-83-63359-81-2.

Szostek Karol, Szostek Roman, Szczególna Teoria Eteru (in Polish), Wydawnictwo Amelia, Rzeszów, Polska, 2015, (www.ste.com.pl), ISBN 978-83-63359-77-5.

[9] Szostek Karol, Szostek Roman, The explanation of the Michelson-Morley experiment results by means universal frame of reference (in English), Journal of Modern Physics, Vol. 8, No. 11, 2017, 1868-1883, ISSN 2153-1196, https://doi.org/10.4236/jmp.2017.811110.

Szostek Karol, Szostek Roman, Wyjaśnienie wyników eksperymentu Michelsona-Morleya przy pomocy teorii z eterem (in Polish), viXra 2017, www.vixra.org/abs/1704.0302.

Szostek Karol, Szostek Roman, Объяснение результатов эксперимента МайкельсонаМорли при помощи универсальной системь отсчета (in Russian), viXra 2018, www.vixra.org/abs/1801.0170.

[10] Szostek Karol, Szostek Roman, The derivation of the general form of kinematics with the universal reference system (in English), Results in Physics, Volume 8, 2018, 429-437, ISSN: 2211-3797, https://doi.org/10.1016/j.rinp.2017.12.053.

Szostek Karol, Szostek Roman, Wyprowadzenie ogólnej postaci kinematyki z uniwersalnym uktadem odniesienia (in Polish), viXra 2017, www.vixra.org/abs/1704.0104.

Szostek Karol, Szostek Roman, Вывод общего вида кинематики с универсальной системой omcчema (in Russian), viXra 2018, www.vixra.org/abs/1806.0198.

[11] Szostek Karol, Szostek Roman, Kinematics in Special Theory of Ether (in English), Moscow University Physics Bulletin, Vol. 73, № 4, 2018, 413-421, ISSN 0027-1349, https://doi.org/10.3103/S0027134918040136. 
Derivation of all linear transformations that meet the results of Michelson-Morley's experiment and discussion of the relativity basics - Szostek Roman

Szostek Karol, Szostek Roman, Kinematyka w Szczególnej Teorii Eteru (in Polish), viXra 2019, www.vixra.org/abs/1904.0195.

Szostek Karol, Szostek Roman, Кинематика в Специальной Теории Эфира (in Russian), Вестник Московского Университета. Серия 3. Физика и Астрономия, Vol. 73, № 4, 2018, 413-421, ISSN 0579-9384.

[12] Szostek Roman, Derivation method of numerous dynamics in the Special Theory of Relativity (in English), Open Physics, Vol. 17, 2019, 153-166, ISSN: 2391-5471, https://doi.org/10.1515/phys-2019-0016.

Szostek Roman, Metoda wyprowadzania licznych dynamik w Szczególnej Teorii Względności (in Polish), viXra 2017, www.vixra.org/abs/1712.0387.

Szostek Karol, Szostek Roman, Метод вывода многочисленных динамик в Специальной Теории Относительности (in Russian), viXra 2018, www.vixra.org/abs/1801.0169.

[13] Szostek Roman, The original method of deriving transformations for kinematics with a universal reference system (in English), viXra 2018, www.vixra.org/abs/1804.0045.

Szostek Roman, Oryginalna metoda wyprowadzania transformacji dla kinematyk $z$ uniwersalnym uktadem odniesienia (in Polish), viXra 2017, www.vixra.org/abs/1710.0103, www.vixra.org/abs/1710.0103.

[14] Tangherlini Frank R., The Velocity of Light in Uniformly Moving Frame, A Dissertation. Stanford University, 1958 (reprint in The Abraham Zelmanov Journal, Vol. 2, 2009, ISSN 1654-9163). 